\title{
THE LOCAL MAXIMAL SUBGROUPS OF EXCEPTIONAL GROUPS OF LIE TYPE, FINITE AND ALGEBRAIC
}

\author{
ARJEH M. COHEN, MARTIN W. LIEBECK, JAN SAXL, \\ and GARY M. SEITZ
}

[Received 18 May 1990—Revised 24 November 1990]

\section{Introduction}

Following the classification of finite simple groups, one of the major problems in finite group theory is the determination of the maximal subgroups of the almost simple groups-that is, of the groups $X$ such that $L \leqslant X \leqslant$ Aut $L$ for some non-abelian finite simple group $L$. In the investigation of the maximal subgroups $M$ of $X$, the analysis is often divided into two parts: the local case, in which $M=N_{X}(E)$ for some elementary abelian subgroup $E$ of $X$; and the non-local case, in which the socle of $M$ is a direct product of non-abelian simple groups. In this paper we determine the local maximal subgroups of the finite exceptional groups of Lie type in the families $G_{2}, F_{4}, E_{6}, E_{7}, E_{8},{ }^{2} G_{2},{ }^{2} F_{4}$ and ${ }^{2} E_{6}$. (The maximal subgroups of the other families of exceptional groups, ${ }^{2} B_{2}$ and ${ }^{3} D_{4}$, can be found in $[29,18]$.) It is a consequence of our main result, Theorem 1 (stated in $\S 1$ ), together with the results of [23] and work on the other simple groups discussed in [21, I-III], that the local maximal subgroups of the almost simple groups are all explicitly known, apart from the 2-locals of the sporadic groups $B M$ and $M$. Theorem 1 is used in the proof of [22, Theorem 2], where the study of maximal subgroups of finite exceptional groups of Lie type is reduced to the case of almost simple subgroups. We remark that the 'maximal local subgroups' of $X$ form a larger class than the 'local maximal subgroups', and we make no attempt to determine the former class.

For most of our proof of Theorem 1 we work in the simple algebraic group $G$ corresponding to the finite exceptional group $L$. Our methods also yield the determination of the local maximal subgroups of simple algebraic groups of exceptional type over algebraically closed fields. The results are stated in Theorem 2 (the positive-dimensional subgroups) and Theorem 3 (the zerodimensional subgroups). Theorem 2 is used in [22, Theorem 1], which determines all positive-dimensional maximal subgroups of $G$.

One of the local subgroups occurring in the conclusion of Theorem 1 is a subgroup $5^{3} \cdot \mathrm{SL}_{3}(5)$ of $E_{8}\left(p^{a}\right)$ (see Table 1 ). This turns out to be non-maximal when $p=2$, because it lies in a subgroup $L_{4}(5)$ of $E_{8}(4)$ in that case. This embedding $L_{4}(5)<E_{8}(4)$, which may be of independent interest, is exhibited in $\S 5$.

Finally, we remark that our proofs are all independent of the classification of finite simple groups.

\footnotetext{
This work was supported by the Science and Engineering Research Council of Great Britain, through Visiting Research Fellowships for the first and fourth authors. The fourth author also acknowledges the support of an NSF grant.

1991 Mathematics Subject Classification: 20E28, 20D06, 20G40.

Proc. London Math. Soc. (3) 64 (1992) 21-48.
} 


\section{Statement of results and notation}

Let $L$ be a finite exceptional simple group of Lie type over $\mathbf{F}_{q}$, where $q=p^{a}$ and $p$ is prime. As described in [27], there is a simple adjoint algebraic group $G$ over the algebraic closure of $\mathbf{F}_{q}$, and a surjective endomorphism $\sigma$ of $G$ such that $L=O^{p^{\prime}}\left(G_{\sigma}\right)$. Also $G_{\sigma}=\operatorname{Inndiag}(L)$, the group generated by all inner and diagonal automorphisms of $L$.

Let $X$ be a group such that $L \leqslant X \leqslant$ Aut $L$, and let $M$ be a local maximal subgroup of $X$. Then $M=N_{X}(E)$ for some elementary abelian $r$-subgroup $E$ of $X$ with $r$ prime. Choosing $E$ minimal, we may assume that

$M$ normalizes no proper non-trivial subgroup of $E$.

Assume that $E \leqslant G_{\sigma}$ (the case where $E \$ G_{\sigma}$ is discussed in the note after Theorem 1 below). If $r=p$ then by [5,3.12] (see also [8]), $M$ lies in a parabolic subgroup of $X$. Otherwise, we may take it that one of the following holds:

(I) $E$ lies in a $\sigma$-stable maximal torus of $G$; or

(II) $M$ normalizes no non-trivial subgroup of a torus of $G_{\sigma}$.

In Case (I), let $D=C_{G}(E)^{0}$. Then $D$ is a $\sigma$-stable closed connected reductive subgroup of $G$ containing a maximal torus, and $M=N_{X}\left(D_{\sigma} \cap L\right)$. In the situation of the previous sentence, we say that $M$ is a subgroup of maximal rank in $X$ (and also that $D$ is a subgroup of maximal rank in $G$ ). The subgroup $D$ has a root system relative to the maximal torus which is a subsystem of the root system of $G$. The possibilities for such subsystems are given in Tables A and B of [23, §2], and the results of [23] include a complete determination of the maximal subgroups of maximal rank in exceptional groups of Lie type.

Theorem 1 determines all the subgroups $M$ in Case (II).

THeorem 1. Let $L, X, G$ be as above, and let $M=N_{X}(E)$ be a local maximal subgroup of $X$, with $E \leqslant G_{\sigma}, E$ an elementary abelian $r$-group. Then either

(I) $M$ is a parabolic subgroup or a subgroup of maximal rank (determined in [23]), or

(II) the pair $(L, E)$ is as in Table 1 ; in each case $r \neq p$ and there is just one $G_{\sigma}$-conjugacy class of such subgroups $E$.

In Table 1 we use the notation $E_{6}^{\varepsilon}(q)$ for $E_{6}(q)$ if $\varepsilon=+1$, and ${ }^{2} E_{6}(q)$ if $\varepsilon=-1$. Also for a prime $r$, we write just $r^{e}$ for an elementary abelian group of that order.

TABLE 1

\begin{tabular}{|c|c|c|c|c|}
\hline$L$ & $E$ & $C_{G_{o}}(E)$ & $N_{G_{\sigma}}(E) / C_{G_{\sigma}}(E)$ & Conditions \\
\hline $\begin{array}{l}G_{2}(p) \\
{ }^{2} G_{2}(3)^{\prime} \\
F_{4}(p) \\
E_{6}^{\varepsilon}(p) \\
E_{7}(q) \\
E_{8}(p)\end{array}$ & $\begin{array}{l}2^{3} \\
2^{3} \\
3^{3} \\
3^{3} \\
2^{2} \\
2^{5}\end{array}$ & $\begin{array}{c}E \\
E \\
E \\
\text { special, of order } 3^{6} \\
E \times\left(P \Omega_{8}^{+}(q) \cdot 2^{2}\right) \\
\text { special, of order } 2^{15}\end{array}$ & $\begin{array}{l}\mathrm{SL}_{3}(2) \\
Z_{7} \\
\mathrm{SL}_{3}(3) \\
\mathrm{SL}_{3}(3) \\
S_{3} \\
\operatorname{SL}_{5}(2)\end{array}$ & $\begin{array}{c}p \geqslant 5 \\
\varepsilon= \pm 1,3 \mid p-\varepsilon, p \geqslant 5 \\
P \Omega_{8}^{+}(q) \cdot 2^{2}=\operatorname{Inndiag}\left(D_{4}(q)\right)\end{array}$ \\
\hline$E_{8}\left(p^{a}\right)$ & $5^{3}$ & $E$ & $\mathrm{SL}_{3}(5)$ & $p \neq 2,5, \quad a=\left\{\begin{array}{l}1, \text { if } 5 \mid p^{2}-1 \\
2, \text { if } 5 \mid p^{2}+1\end{array}\right.$ \\
\hline $\begin{array}{l}{ }^{2} E_{6}(2) \\
E_{7}(3)\end{array}$ & $\begin{array}{l}3^{2} \\
2^{2}\end{array}$ & $\begin{array}{l}E \times G_{2}(2) \\
E \times F_{4}(3)\end{array}$ & $\begin{array}{c}N_{L}(E)=U_{3}(2) \times G_{2}(2) \\
N_{L}(E)=L_{2}(3) \times F_{4}(3)\end{array}$ & \\
\hline
\end{tabular}


REMARK. In the maximal rank case in (I), our proof in fact shows that $M$ normalizes a non-trivial subgroup of a torus of $G_{\sigma}$ (see Lemma 2.2).

Constructions of the subgroups in Table 1 can be found in the proof of Theorem 1 in $\S 2$. It is of interest to note that the condition that $p \neq 2$, when $E$ is $3^{3}$ or $5^{3}$ and $L$ is $F_{4}(p)$ or $E_{8}\left(p^{a}\right)$, occurs because of the embeddings $L_{4}(3)<F_{4}(2)$ and $L_{4}(5)<E_{8}(4)$; the subgroups $L_{4}(3)$ and $L_{4}(5)$ contain $N_{L}(E)$ in each case. The embedding $L_{4}(3)<F_{4}(2)$ is known $[\mathbf{1 1}, \mathbf{2 5}]$, but the fact that $L_{4}(5)<E_{8}(4)$ is new, and we give a proof in $\S 5$.

Note. In Theorem 1 we assume that $E \leqslant G_{\sigma}$. When $E \$ G_{\sigma}$, we have $E \cap G_{\sigma}=1$ by (*), and so $M \cap L=C_{L}(\alpha)$ for some automorphism $\alpha \epsilon$ (Aut $L) \backslash G_{\sigma}$ of prime order $r$. Then $\alpha$ is a field, graph-field or graph automorphism (see $[16, \S 7])$. The conjugacy classes of such automorphisms are known, by [16, $\S 7 ; 3, \S 19]$ and Proposition 2.7 of this paper. The centralizers arising are subgroups of the same type as $L$ over a maximal subfield of $\mathbf{F}_{q}$, subgroups ${ }^{2} G_{2}(q)$, ${ }^{2} F_{4}(q),{ }^{2} E_{6}\left(q^{\frac{1}{2}}\right)$ in $G_{2}(q), F_{4}(q), E_{6}(q)$, and subgroups $C_{4}(q)$ (with $q$ odd) and $F_{4}(q)$ in $E_{6}^{\varepsilon}(q)$.

Next we turn to algebraic groups. We prove Theorems 2 and 3 , which determine all local subgroups (that is, normalizers of finite abelian subgroups) of simple algebraic groups of exceptional type over algebraically closed fields, subject to certain maximality conditions. Theorem 2 handles the subgroups of positive dimension and Theorem 3 those of dimension zero.

TheOREM 2. Let $G$ be a simple adjoint algebraic group of exceptional type $\left(G_{2}, F_{4}, E_{6}, E_{7}\right.$ or $\left.E_{8}\right)$ over an algebraically closed field of characteristic l. Let $S$ be a subgroup of Aut $G$ such that $D=(S \cap G)^{0}$ is a non-trivial closed connected subgroup of $G$, and assume that

(1) $N_{G}(D) / D$ is finite,

(2) for some prime $r, O_{r}\left(C_{G}(D)\right) \neq 1$, and

(3) $D$ is maximal among closed connected $S$-invariant subgroups of $G$.

Then either $D$ is parabolic or a subgroup of maximal rank, or $G=E_{7}, l \neq 2$, $D=D_{4}$ and $N_{G}(D)=\left(2^{2} \times D_{4}\right) . S_{3}\left(\right.$ with $C_{G}(D)=2^{2}$ as in Table 1$)$.

Theorem 3. Let $G$ be as in Theorem 2, and suppose $A$ is a subgroup of $G$ satisfying the following conditions:

(a) $A$ is an elementary abelian $r$-group, where $r$ is prime and $r \neq l$,

(b) $N_{G}(A)$ is finite,

(c) $N_{G}(A)$ normalizes no proper non-trivial subgroup of $A$,

(d) $N_{G}(A)$ is maximal with respect to (a), (b) and (c),

(e) there is no proper non-trivial connected $N_{G}(A)$-invariant subgroup of $G$.

Then $A$ is given in Table 2, and is uniquely determined up to G-conjugacy.

Theorem 3 generalizes to arbitrary characteristic a result stated for characteristic zero in [1]. We give the proof, which runs along the same lines as that of Theorem 1 , in $\S 4$. The subgroups $A$ in Table 2 are called Jordan subgroups of $G$ in [1], and a result similar to Theorem 3 concerning Jordan subgroups is proved in $[7]$. 
TABLE 2

\begin{tabular}{cll}
\hline$G$ & $A$ & \multicolumn{1}{c}{$N_{G}(A)$} \\
\hline$G_{2}$ & $2^{3}$ & $2^{3} \cdot \mathrm{SL}_{3}(2)$ \\
$F_{4}$ & $3^{3}$ & $3^{3} \cdot \mathrm{SL}_{3}(3)$ \\
$E_{6}$ & $3^{3}$ & $3^{3+3} \cdot \mathrm{SL}_{3}(3)$ \\
$E_{8}$ & $2^{5}$ & $2^{5+10} \cdot \mathrm{SL}_{5}(2)$ \\
& $5^{3}$ & $5^{3} \cdot \mathrm{SL}_{3}(5)$ \\
\hline
\end{tabular}

Corollary. Let $G$ be as in Theorem 2.

(i) The local maximal subgroups of $G$ of positive dimension are those given in Theorem 2.

(ii) Suppose $H$ is a finite local subgroup of $G$ which is contained in no proper closed connected subgroup of $G$. Then $H$ is one of the groups $N_{G}(A)$ in Table 2.

The corollary is immediate from Theorems 2 and 3.

The actions of the local subgroups $N_{G}(A)$ in Table 2 on the Lie algebra $L(G)$ of $G$ yield interesting orthogonal decompositions of $L(G)$, which are studied in $[6,19]$.

The layout of the rest of the paper is as follows. Sections 2, 3 and 4 contain the proofs of Theorems 1,2 and 3 , respectively. Finally, in $\S 5$ we exhibit the embedding $L_{4}(5)<E_{8}(4)$.

\section{Proof of Theorem 1}

Assume the hypotheses of Theorem 1. Thus $G$ is a simple adjoint group of exceptional type, $L=O^{p^{\prime}}\left(G_{\sigma}\right)$ is a simple group of Lie type over $\mathbf{F}_{q}, q=p^{a}$, and $X$ is a group with $L \leqslant X \leqslant$ Aut $L$. Also $M=N_{X}(E)$ is a non-parabolic local maximal subgroup of $X$ normalizing no non-trivial subgroup of a torus of $G_{\sigma}$, and also normalizing no non-trivial proper subgroup of $E$, where $E$ is an elementary abelian $r$-subgroup of $G_{\sigma}$ consisting of semisimple elements. Notice that we are not excluding the possibility that $M$ is of maximal rank here; in fact, Lemma 2.2 rules this out (see the Remark after Theorem 1).

We introduce some further notation. Let $\hat{G}$ be the simply connected cover of $G$, let $\hat{E}$ be the full preimage of $E$ in $\hat{G}$, and let $\hat{g}$ be a preimage of an element $g \in G$. Write also $E^{\#}=E \backslash\{1\}$, and $W=W(G)$, the Weyl group of $G$, and denote by $T_{i}$ a torus of rank $i$ in $G$.

If $K$ is a connected reductive subgroup of $G$, define a homogeneous factor of $K$ to be the product of all the simple connected normal subgroups of $K$ in a single (Aut $G$ )-conjugacy class; if $Z(K)^{0} \neq 1$, define $Z(K)^{0}$ also to be a homogeneous factor. Thus $K$ is the commuting product of its homogeneous factors.

Note that Aut $L$ is generated by inner, diagonal, field and graph automorphisms (see $[9,28]$ ), all of which extend to automorphisms of the abstract group $G$ which commute with $\sigma$. Thus there is a subgroup $\tilde{X}$ of $C_{\text {Aut } G}(\sigma)$ such that $X=\tilde{X} /\langle\sigma\rangle$, and so $X$ acts on the set of $\sigma$-stable subsets of $G$. For a $\sigma$-stable subset $Y$, we write $N_{X}(Y)$ for the stabilizer in $X$ of $Y$.

We now embark upon the proof of Theorem 1 . This is carried out in a series of lemmas (2.1 to 2.17). Lemmas 2.1 to 2.9 are concerned with restricting the structure of $C_{G}(e)$ for $e \in E^{\#}$. The remaining lemmas deal separately with the various possibilities for $G$ and $r$. 
Lemma 2.1. Suppose that $M\langle\sigma\rangle$ normalizes a non-trivial, proper, connected, semisimple subgroup $K$ of $G$. Then one of the following holds:

(i) $[K, E]=1$;

(ii) $q=3$ and $K$ has components of type $A_{1}$ such that if $C$ is the product of these components then $E \triangleleft O^{p^{\prime}}\left(C_{\sigma}\right) \cong L_{2}(3)^{x}$, a direct product of $x$ copies of $L_{2}(3)$; in particular, the rank of $E$ is even;

(iii) $q=2$ and $K$ has components of type $A_{2}$ such that if $C$ is the product of these components then $E \triangleleft O^{p^{\prime}}\left(C_{\sigma}\right) \cong U_{3}(2)^{y}$ for some $y \geqslant 1$.

Proof. As $K_{\sigma} \neq 1$, by the maximality of $M$ we have $M=N_{X}\left(K_{\sigma}\right)=N_{X}(E)$. Thus $K_{\sigma} \leqslant M$ and $E \leqslant N\left(K_{\sigma}\right)$. It follows that

$$
\left[K_{\sigma}, E\right] \triangleleft K_{\sigma}, \quad\left[K_{\sigma}, E\right] \leqslant K_{\sigma} \cap E .
$$

Assume first that every factor of $K_{\sigma}^{\prime}$ is quasisimple, where $K_{\sigma}^{\prime}$ means $\left(K_{\sigma}\right)^{\prime}$. We show that conclusion (i) holds. Since $\left[K_{\sigma}^{\prime}, E\right] \triangleleft K_{\sigma}^{\prime}$, we deduce that $\left[K_{\sigma}^{\prime}, E\right] \leqslant$ $Z\left(K_{\sigma}^{\prime}\right)$, so that $\left[K_{\sigma}^{\prime}, E, K_{\sigma}^{\prime}\right]=\left[E, K_{\sigma}^{\prime}, K_{\sigma}^{\prime}\right]=1$. By the three-subgroup lemma, $\left[K_{\sigma}^{\prime}, E\right]=1$. Now let $e \in E^{\#}$. Then $K_{\sigma}^{\prime} \leqslant C_{K}(e)$.

We claim that $C_{K}(e)$ is reductive. For if not, let $Q=R_{u}\left(C_{K}(e)\right)$, the unipotent radical of $C_{K}(e)$. Then $K_{\sigma}^{\prime} \leqslant N_{K}(Q) \leqslant P$, where $P$ is the canonical parabolic subgroup of $K$ determined by $Q$ as in [5]. Moreover $P$ is $\sigma$-stable and $P_{\sigma}$ is a parabolic subgroup of $K_{\sigma}$. But $K_{\sigma}^{\prime}$ normalizes $R_{u}(P)_{\sigma}$ and $R_{u}(P)_{\sigma} \leqslant O^{p^{\prime}}\left(K_{\sigma}\right)=$ $K_{\sigma}^{\prime}$, so $R_{u}(P)_{\sigma} \triangleleft K_{\sigma}^{\prime}$, a contradiction. Thus $C_{K}(e)$ is reductive, as claimed. Now $K_{\sigma}^{\prime}=\left(C_{K}(e)_{\sigma}\right)^{\prime}$, so, in particular,

$$
\left|K_{\sigma}^{\prime}\right|_{p}=\left|C_{K}(e)_{\sigma p}^{\prime}\right|_{p}
$$

Since $\left|K_{\sigma}^{\prime}\right|_{p}$ is $q^{n}$, where $n$ is the number of positive roots in the root system of $K$, we deduce that $C_{K}(e)=K$. Hence $[K, E]=1$, giving conclusion (i).

Now suppose that (i) fails. Then some factor of $K_{\sigma}$ is not quasisimple, so is of type $A_{1}(2), A_{1}(3),{ }^{2} A_{2}(2)$ or ${ }^{2} B_{2}(2)$. If there is a factor $A_{1}(2)$ or ${ }^{2} B_{2}(2)$ then the product $A$ of all such factors is $M$-invariant, so $M \leqslant N\left(O_{r}(A)\right)$ where $r$ is 3 or 5 ; but $O_{r}(A)$ intersects each factor in a cyclic group, and so lies in a maximal torus of $G$, contrary to our hypothesis on $M$. If there is a factor $A_{1}(3)$ or ${ }^{2} A_{2}(2)$ of the form $\mathrm{SL}_{2}(3)$ or $\mathrm{SU}_{3}(2)$ then $M$ normalizes the product of the centres of all such factors, which again lies in a torus. Thus $K$ must have components of type $A_{1}$ or $A_{2}$, such that if $C$ is the product of these components then $O^{p^{\prime}}\left(C_{\sigma}\right) \cong L_{2}(3)^{x}$ or $U_{3}(2)^{y}$ for some positive integers $x, y$. Moreover, if $\left[C_{\sigma}, E\right]=1$ then $\left|C_{\sigma}\right|_{p}=$ $\left|C_{C}(e)_{\sigma}\right|_{p}$, and hence as above, $[C, E]=1$; consequently $[K, E]=1$, which is false. So $\left[C_{\sigma}, E\right] \neq 1$. Since $M$ normalizes $C_{\sigma}$ and $E$, we have $1 \neq\left[C_{\sigma}, E\right] \leqslant$ $C_{\sigma} \cap E$, and hence $E \leqslant C_{\sigma}$ as $M$ normalizes no proper subgroup of $E$. Thus (ii) or (iii) holds.

LEMMA 2.2. Suppose that $M\langle\sigma\rangle$ normalizes a connected subgroup $D$ of $G$, such that $D$ is normalized by a maximal torus of $G$. Then $D$ is 1 or $G$.

Proof. Suppose that $D$ is not 1 or $G$, and take $D$ maximal with respect to the hypotheses of the lemma. Let $T$ be a maximal torus in $N_{G}(D)$. If $Q=R_{u}(D) \neq 1$ then $M\langle\sigma\rangle \leqslant N_{G}(Q) \leqslant P$, where $P$ is the canonical parabolic subgroup of $G$ determined by $Q$ as in [5]. But then $M$ normalizes the parabolic $P_{\sigma}$, so $M$ must be parabolic, a contradiction. 
Thus $D$ is reductive, so $D=D^{\prime} Z$ with $D^{\prime}$ semisimple and $Z=Z(D)^{0}$. The maximality of $D$ implies that $D=\left(D C_{G}(D)\right)^{0}$. We claim that $D T \leqslant D C_{G}(D)$ : for $D T$ is reductive and so corresponds to a subsystem of roots relative to $T$, from which the claim is clear. Thus $T \leqslant D$, and hence $Z=C_{T}(D)^{0}$. Since $M$ normalizes $Z$, our hypothesis on $M$ forces $Z_{\sigma}=1$. Moreover, we may take $T$ to be $\sigma$-stable, by $[26, I, 2.9]$.

Suppose that $D^{\prime} \neq 1$. Since $T \leqslant D$, components $A_{1}$ and $A_{2}$ of the root system of $D^{\prime}$ (relative to $T$ ) are generated by root subgroups of $G$, so cannot have $\sigma$-fixed point groups of the form $L_{2}(3)$ or $U_{3}(2)$. Thus by Lemma 2.1 we have $\left[D^{\prime}, E\right]=1$. Note also that $Z \neq 1$, since otherwise $T \leqslant D=D^{\prime}$, giving $E \leqslant$ $C_{G}\left(D^{\prime}\right)_{\sigma} \leqslant C_{G}(T)_{\sigma}=T_{\sigma}$, contrary to hypothesis. Also $Z(D) \leqslant C(T)=T$. We have now

$$
D=D^{\prime} Z, \quad D^{\prime} \neq 1, \quad Z \neq 1 .
$$

We next claim that $C_{G}\left(D^{\prime}\right)=Z\left(D^{\prime}\right) Z$. To see this, let $D^{\prime}=H_{1} \ldots H_{m}$ be the expression for $D^{\prime}$ as a commuting product of its homogeneous factors $H_{i}$ (see the beginning of this section for the definition). By the maximality of $D$, for each $i$, $C_{G}\left(H_{i}\right)^{0}$ is the product of those $H_{j}$ with $j \neq i$, and also $C_{G}(Z)^{0}=D$. Moreover, the rank of $D^{\prime}$ is less than the rank of $G$, as $Z \neq 1$. Let $\Delta$ be the root system of $D^{\prime}$. The possibilities for $\Delta$ satisfying the above conditions are not hard to determine, using the lists of all closed subsystems of the root system of $G$ given in [10, Tables 7-11]. Indeed, the possibilities are as follows:

\begin{tabular}{c|ccccc}
$G$ & $G_{2}$ & $F_{4}$ & $E_{6}$ & $E_{7}$ & $E_{8}$ \\
\hline$\Delta$ & none & $B_{3}(p$ odd $)$ & $D_{5}, D_{4}, 4 A_{1}, 2 A_{1}+A_{3}$ & $E_{6}, A_{6}, 3 A_{2}$ & $D_{7}, A_{7}, 2 A_{3}$
\end{tabular}

Assume that $G \neq F_{4}$. Then $C_{W}(\Delta)=1$ (see [10, Tables 7-11]). Since $N_{G}(D) \leqslant$ $D N_{G}(T)$, this forces $C_{G}\left(D^{\prime}\right)=C_{T}\left(D^{\prime}\right)=Z\left(D^{\prime}\right) Z$, as claimed. And if $G=F_{4}$, $\Delta=B_{3}$ and $p$ is odd, then $M$ normalizes $Z(D) \cong Z_{2}$, contrary to hypothesis. This establishes the claim. It follows that $E \leqslant C_{G}\left(D^{\prime}\right)_{\sigma}=\left(Z\left(D^{\prime}\right) Z\right)_{\sigma} \leqslant T_{\sigma}$, a contradiction.

We have now shown that $D^{\prime}=1$, and so $D=T$. Consequently $M$ normalizes $T_{\sigma}$, so by hypothesis we must have $T_{\sigma}=1$. By [26, II, 1.7], $\left|T_{\sigma}\right|=f(q)$, where $f$ is the characteristic polynomial of some $w \in W$ acting on the associated Euclidean space. It follows that $w=1$ and $q=2$, so that $M \cap G_{\sigma} \cong W$. Now for $G \neq G_{2}$, $E \triangleleft W$ forces $r=q=2$, a contradiction. And if $G=G_{2}$ then $L=G_{2}(2)$ and $M$ is clearly non-maximal. This completes the proof.

Lemma 2.3. Suppose that $K$ is a connected subgroup of $G$ normalized by $M\langle\sigma\rangle$. Then $K$ is semisimple and $Z(K)=1$. In particular, $C_{G}(E)^{0}$ is semisimple with trivial centre.

Proof. First, $K$ is reductive: for if not, we can use [5], as at the beginning of the proof of Lemma 2.2 , to show that $M$ is parabolic, a contradiction. Thus $K=K^{\prime} Z(K)$ with $K^{\prime}$ semisimple and $Z(K)$ contained in a torus. We must have $Z(K)=1$ by Lemma 2.2 . The result follows.

LeMma 2.4. (i) If $e \in E^{\#}$ then $E=\left\langle e^{G} \cap E\right\rangle$.

(ii) $r$ is 2, 3 or 5. Moreover, $r$ is 5 only if $G=E_{8}$.

(iii) The rank $m(E)$ of $E$ is at least 2. Further, if $(G, r)$ is not $\left(E_{6}, 3\right)$ or $\left(E_{7}, 2\right)$ then $m(E) \geqslant 3$. 
Proof. Part (i) is immediate from the hypothesis that $M$ normalizes no proper non-trivial subgroup of $E$. By assumption, $E$ does not lie in a torus of $G$. Hence (ii) follows from [26, II, 5.8 and 5.11], as the torsion primes for $G$ (see [26, I, 4.4]) are 2, 3 and 5, with 5 occurring only for $E_{8}$. Finally, for (iii), suppose that $E=\langle e, f\rangle$ and $(G, r)$ is not $\left(E_{6}, 3\right)$ or $\left(E_{7}, 2\right)$. Then $C_{G}(e)$ is connected, by [26, II, 4.6]. Hence there is a torus of $C_{G}(e)$ containing $f$ and $e$, again a contradiction. Thus $m(E) \geqslant 3$, as required.

Lemma 2.5. (i) $C_{G}(E)$ contains no normal torus.

(ii) Let $e \in E^{\#}$. Then $C_{G}(e)$ does not contain a central torus. Moreover, if $C_{G}(e)$ contains a normal torus then $E \$ C_{G}(e)^{0}$.

Proof. Part (i) is immediate from Lemma 2.3. Part (ii) follows from (i), since if $T$ is a torus in $Z(C(e))$ then $T \leqslant Z(C(E))$.

The possibilities for the centralizers in $G$ of semisimple elements can be calculated using $[\mathbf{1 6}, 14.1]$ and its proof. Provided $(G, r)$ is not $\left(E_{6}, 3\right)$ or $\left(E_{7}, 2\right)$, $C_{G}(e)$ is connected, and hence has no normal torus by Lemma 2.5(ii). From these observations we deduce:

Lemma 2.6. For $e \in E^{\#}$, the group $C_{G}(e)$ has one of the structures given in Table 3 below. In the table, $w_{2}$ and $w_{3}$ denote elements of $W=W(G)$ of orders 2 and 3 , respectively.

TABLE 3

\begin{tabular}{lccc}
\hline$G$ & $r=2$ & $r=3$ & $r=5$ \\
\hline$G_{2}$ & $A_{1} \circ A_{1}$ & $A_{2}$ & \\
$F_{4}$ & $A_{1} \circ C_{3}$ or $B_{4}$ & $A_{2} \circ A_{2}$ & \\
$E_{6}$ & $A_{1} \circ A_{5}$ & $\left(A_{2} \circ A_{2} \circ A_{2}\right)\left\langle w_{3}\right\rangle$ or $T_{2} D_{4}\left\langle w_{3}\right\rangle$ & \\
$E_{7}$ & $A_{1} \circ D_{6}, A_{7}\left\langle w_{2}\right\rangle$ or $T_{1} E_{6}\left\langle w_{2}\right\rangle$ & $A_{2} \circ A_{5}$ & \\
$E_{8}$ & $A_{1} \circ E_{7}$ or $D_{8}$ & $A_{2} \circ E_{6}$ or $A_{8}$ & $A_{4} \circ A_{4}$ \\
\hline
\end{tabular}

In order to restrict further the possibilities for $C_{G}(e)\left(e \in E^{\#}\right)$, it is convenient to handle first the local subgroups $U_{3}(2) \times G_{2}(2)$ in ${ }^{2} E_{6}(2)$ and $L_{2}(3) \times F_{4}(3)$ in $E_{7}(3)$ given in Table 1 of Theorem 1 . For this we require a proposition concerning graph automorphisms of groups of type $A_{n}$ and $E_{6}$.

Proposition 2.7. Assume that $p$ is odd and that $Y$ is a simple adjoint algebraic group of type $A_{2 n-1}$ or $E_{6}$ over the algebraic closure of $\mathbf{F}_{p}$. Let $\tau$ be the standard involutory graph automorphism of $Y$ given in [9, Chapter 12] (with centralizer $C_{n}$ or $F_{4}$ respectively). Then there are precisely two classes of involutions in $Y \tau$, with representatives $\tau$ and $\tau h$, where $h$ is an involution in $Y$. The connected centralizers of $\tau$, $\tau h$ have types $C_{n}, D_{n}$ if $Y=A_{2 n-1}$, and types $F_{4}, C_{4}$ if $Y=E_{6}$.

Proof. Let $\hat{Y}$ be the simply connected cover of $Y$. Pick $\gamma \in \hat{Y} \tau$. By [27, 7.5], $\gamma$ normalizes a Borel subgroup $B$ of $\hat{Y}$ and a maximal torus $T$ of $B$. Moreover $N_{\hat{Y}\langle\tau\rangle}(T) \cap N_{\hat{Y}\langle\tau\rangle}(B)=T\langle\delta\rangle$ with $\delta$ a conjugate of $\tau$, so we may take $\delta=\tau$ and $\gamma=\tau t$ for some $t \in T$. Further, $T$ is the direct product of 1-dimensional tori $T_{\alpha}$, one for each fundamental root $\alpha$ of $\hat{Y}$. 
Suppose that $Y=E_{6}$. Here $\tau$ interchanges two pairs of groups $T_{\alpha}$ and fixes the other two. Write $T=T_{n} T_{f}$, where $T_{n}$ (respectively, $T_{f}$ ) is the product of those $T_{\alpha}$ not fixed (respectively, fixed) by $\tau$, and corresponding to this decomposition, write $t=t_{n} t_{f}$. Since $\tau t$ has order $2, \tau t$ is conjugate to $\tau t_{f}$ by an element of $T$, so we replace $\tau t$ by $\tau t_{f}$. Now $t_{f}^{2}=1$ and $t_{f}$ lies in the product of the two fixed tori $T_{\alpha}$, so $t_{f}$ is contained in a subgroup $A_{2}$ centralized by $\tau$. All involutions in this $A_{2}$ are conjugate. Thus $\gamma$ is conjugate to either $\tau$ or $\tau t_{\alpha}$, where $t_{\alpha}$ is the involution in a fundamental subgroup $\mathrm{SL}_{2}$ centralized by $\tau$. Conjugating by an element of the Weyl group of $C_{Y}(\tau)$, we see that $\alpha$ can be replaced by the highest root $\alpha_{0}$. Finally, we identify $C_{Y}(\tau)$ and $C_{Y}\left(\tau t_{\alpha_{1}}\right)$. We know that $C_{Y}(\tau) \cong F_{4}$. We now claim that $C_{Y}\left(\tau t_{\alpha_{1}}\right) \cong C_{4}$. First note that $C_{Y}(\tau)$ contains $D=\left(A_{1}\right)^{4}$, and this can be chosen with $t_{\alpha_{0}} \in Z(D)$. So it is clear that $C_{Y}\left(\tau t_{\alpha_{0}}\right)$ contains $D$. Let $\alpha$ be a root of $E_{6}$ and $U_{\alpha}$ the corresponding $T$-root subgroup. If $\alpha \tau=\alpha$ then one checks from the known action of the graph automorphism $\tau$ that $U_{\alpha} \leqslant C_{Y}\left(\tau t_{\alpha_{0}}\right)$ if and only if $t_{\alpha_{0}}$ centralizes $U_{\alpha}$. A direct check of roots shows that such root subgroups $U_{\alpha}$ span $D$. Now suppose that $\alpha \tau \neq \alpha$. One checks from the Chevalley commutator relations that in each case $\left\langle U_{\alpha}, U_{\alpha \tau}\right\rangle=U_{\alpha} \times U_{\alpha \tau}$, and hence $C_{Y}\left(\tau t_{\alpha_{1}}\right) \cap\left\langle U_{\alpha}, U_{\alpha \tau}\right\rangle$ is 1-dimensional. There are 24 pairs of roots interchanged by $\tau$, and hence

$$
\operatorname{dim} L\left(C_{Y}\left(\tau t_{\alpha_{0}}\right)\right) \geqslant 24+\operatorname{dim} L(D)=36 .
$$

On the other hand, the same arguments at the level of the Lie algebra show that $\operatorname{dim}\left(L(Y) \cap C\left(\tau t_{\alpha_{0}}\right)\right)=36$. Thus $C_{Y}\left(\tau t_{\alpha_{1}}\right)$ is a reductive group of dimension 36, and $D$ is a maximal commuting set of fundamental subgroups $\mathrm{SL}_{2}$ (this is already true in $Y$ ). It follows that $C_{Y}\left(\tau t_{\alpha_{1}}\right) \cong C_{4}$, as claimed.

Now let $Y=A_{2 n-1}$, so $\hat{Y}=\mathrm{SL}_{2 n}$. Here it will be convenient to replace $\tau$ by $\tau^{\prime}=h \delta$, where $\delta$ is the inverse-transpose map and $h$ is the $n \times n$ matrix $\left(\delta_{i, n+1-i}\right)$ (that is, the matrix with entries 1 on the opposite diagonal and entries 0 elsewhere). Then $\tau^{\prime} \in \hat{Y} \tau$. Now $\tau^{\prime}$ normalizes the lower triangular group and also the diagonal group $T$. An easy computation shows that any element of $\tau^{\prime} T$ is $T$-conjugate to an element of the form $\tau^{\prime} t$, where $t=\operatorname{diag}\left(1, \ldots, 1, c_{n+1}, \ldots, c_{2 n}\right)$ for some $c_{i} \in \overline{\mathbf{F}}_{p}$. If we also assume that $\tau^{\prime} t$ corresponds to an involution in $Y\langle\tau\rangle$, then $\left(\tau^{\prime} t\right)^{2} \in Z(\hat{Y})$. It then follows that $t=\operatorname{diag}(1, \ldots, 1, c, \ldots, c)$ with $c= \pm 1$. Then $C_{\hat{Y}}\left(\tau^{\prime} t\right)$ is $D_{n}$ or $C_{n}$ according as $c$ is 1 or -1 .

LEMmA 2.8. There are local subgroups $U_{3}(2) \times G_{2}(2)$ in ${ }^{2} E_{6}(2)$, and $L_{2}(3) \times F_{4}(3)$ in $E_{7}(3)$. These subgroups are unique up to $G_{\sigma}$-conjugacy.

Proof. First consider $L=E_{7}(3)$. The adjoint algebraic group $G$ contains three conjugacy classes of involutions, with centralizers $A_{1} D_{6}, A_{7}\left\langle w_{2}\right\rangle$ and $T_{1} E_{6}\left\langle w_{2}\right\rangle$ (see [17]). Let $a$ be an involution in $G_{\sigma}$ with $C_{G}(a)=T_{1} E_{6}\left\langle w_{2}\right\rangle$. Here $w_{2}$ induces a graph automorphism on the $E_{6}$. By Proposition 2.7, we may pick an involution $b \in C_{G_{o}}(a)$ such that $C_{G}(a, b)^{0} \cong F_{4}$. Write $F=C_{G}(a, b)^{0}$. Then $T_{1} \leqslant C_{G}(F)$. Since $C_{G}(b)$ contains $F, b$ must be conjugate to $a$, so $C_{G}(b)=T_{1}^{\prime} E_{6}^{\prime}\left\langle w_{2}^{\prime}\right\rangle$. Then $F \leqslant E_{6}^{\prime}$ and so $C_{G}(F)$ contains $\left\langle T_{1}, T_{1}^{\prime}\right\rangle$. We claim that $C_{G}(F)$ is reductive. For otherwise, $F C_{G}(F)$ lies in a parabolic subgroup $P$ of $G$. Since $P$ contains $F \cong F_{4}$, $P$ must be an $E_{6}$-parabolic with $F$ fixing a 1-space of the unipotent radical. But $F$ also fixes a 1-space of the unipotent radical of the opposite parabolic, contradicting the fact that $C_{G}(F)^{0} \leqslant P$. This proves the claim. Also $C_{G}(F)^{0} \cap C_{G}(a)=T_{1}$, so 
$C_{G}(F)^{0}$ has rank at most 1 , and so $C_{G}(F)^{0} \cong A_{1}$. Thus we have constructed a subgroup $A_{1} \times F_{4}$ in $G=E_{7}$. The subgroup $A_{1}$ here is of adjoint type, as $A_{1} \times F_{4}$ does not centralize any involution. Taking fixed points under $\sigma$ then, we have a subgroup $L_{2}(3) \times F_{4}(3)$ of $E_{7}(3)$. This subgroup is $N_{L}(\langle a, b\rangle)$. Since the above proof shows that $\langle a, b\rangle$ is unique up to $G_{\sigma}$-conjugacy, the result is proved for this case.

The argument for $L={ }^{2} E_{6}(2)$ is similar. Here we take an element $a \in L$ of order 3 with $C_{G}(a)=T_{2} D_{4}\left\langle w_{3}\right\rangle$. Choosing $b=w_{3}$, we find that $C_{G}(a, b)^{0}=G_{2}$, and $a, b$ are conjugate. Further, if $T_{2}^{\prime}$ is the centre of $C_{G}(b)^{0}$, then $C_{G}\left(G_{2}\right)$ contains $\left\langle T_{2}, T_{2}^{\prime}\right\rangle$, and we calculate as above that $C_{G}\left(G_{2}\right)^{0}=A_{2}$. Thus we obtain a subgroup $A_{2} \times G_{2}$ in $E_{6}$, and, taking fixed points, a subgroup $U_{3}(2) \times G_{2}(2)$ in ${ }^{2} E_{6}(2)$. Uniqueness follows as before.

For the remainder of the proof we assume that $N_{L}(E)$ is not one of the subgroups given in Lemma 2.8 .

Lemma 2.9. (i) Suppose that $Z(\hat{G})$ has order $r$. Then $\hat{E}$ (the preimage of $E$ in $\hat{G}$ ) is either elementary abelian or extraspecial.

(ii) For $e \in E^{\#}, C_{G}(e)^{0}$ is semisimple.

Proof. Now $\hat{E}$ is the commuting product of an abelian group and an extraspecial group, and $M$ normalizes $Z(\hat{E})$. Thus our hypothesis on $M$, together with Lemma 2.4(i), implies Part (i).

For (ii), assume that $e \in E^{\#}$ is such that $C_{G}(e)^{0}$ is not semisimple. Then $C_{G}(e)^{0}=T D$, a commuting product of a non-trivial torus $T$ and a semisimple group $D$. By Lemma 2.6, there are precisely two possibilities: $T D=T_{2} D_{4}$ with $(G, r)=\left(E_{6}, 3\right)$, and $T D=T_{1} E_{6}$ with $(G, r)=\left(E_{7}, 2\right)$. By Lemma 2.5(ii), we have $E \$ C_{G}(e)^{0}$, and so Part (i) implies that $\hat{E}$ is extraspecial. Thus for each $f \in E^{\#}$ there is an element $\hat{z} \in Z(\hat{G})^{\#}$ such that $\hat{f}$ is $\hat{E}$-conjugate to $\hat{f} \hat{z}$. Since the order of the multiplier of $D$ is not divisible by $r$, it follows that $E \cap D=1$, and hence $|E|=r^{2}$. Write $E=\langle e, f\rangle$. Define $Y=C_{G}(E)^{0}$ and $K=C_{G}(Y)^{0}$. Then $Y$ and $K$ are both semisimple by Lemma 2.3 , and we have $Y \leqslant D, T \leqslant K$. Moreover $Y \neq 1$, since $f$ induces an element in the coset of a graph automorphism of $D$. Thus $K<G$.

Suppose first that $[K, E]=1$. Then $K \leqslant C_{G}(E)^{0}=Y$ and so $K \leqslant Z(Y)$, which is absurd as $K$ and $Y$ are semisimple.

Thus $[K, E] \neq 1$, so by Lemma $2.1, L$ is $E_{7}(3)$ or $E_{6}^{\varepsilon}(2)$ and $K_{\sigma}$ has factors $L_{2}(3)$ or $U_{3}(2)$, respectively. Since $\hat{E}$ is extraspecial, the multiplier of $L$ has order divisible by $r$, so when $q=2$ we have $L={ }^{2} E_{6}(2)$. Consider $L=E_{7}(3)$. Now $C_{G}(e)=T_{1} E_{6}\left\langle w_{2}\right\rangle$ and $f$ induces an element in the coset of a graph automorphism of $D=E_{6}$. Hence by Proposition 2.7, $Y=C_{G}(E)^{0}$ is $C_{4}$ or $F_{4}$. Moreover, $K \cap C_{G}(e)=T_{1}$, so as $K_{\sigma}$ has a factor $L_{2}(3)$, we must have $K=A_{1}$ and $K_{\sigma}^{\prime}=L_{2}(3)$. Thus $E=\left(Z_{2}\right)^{2} \triangleleft K_{\sigma}$. If $Y=C_{4}$ then $E$ is not fused in $G$ (see the proof of Lemma 2.15 below, third paragraph), which is absurd. Thus $Y=F_{4}$ and $N_{L}(E)=L_{2}(3) \times F_{4}(3)$. This is conjugate to the subgroup of Lemma 2.8, which we have excluded by assumption. An entirely similar argument for $L={ }^{2} E_{6}(2)$ (using here $[\mathbf{1 6}, 9.1]$ for the classes of graph automorphisms of $D$ of order 3 ), shows that $N_{L}(E)$ is the subgroup of Lemma 2.8 in this case also. 
In the remaining lemmas we deal separately with the various possibilities for $G$ and $r$.

LEMMA 2.10. (i) If $G=G_{2}$ then $r=2$ and $N_{G}(E)=2^{3} \cdot \mathrm{SL}_{3}(2)$.

(ii) Let $G$ be $F_{4}$ or $E_{8}$ with $r=3$ or 5 , respectively. Then $N_{G}(E)=r^{3} \cdot \mathrm{SL}_{3}(r)$.

In both cases (i) and (ii), $L$ and $N_{G_{\sigma}}(E)$ are as in Table 1 , and $E$ is determined uniquely up to $G_{\sigma}$-conjugacy.

Proof. Fix $e \in E^{\#}$. First assume that $G=G_{2}$ and $r=3$. Then $E \leqslant C_{G}(e) \cong \mathrm{SL}_{3}$ by Lemma 2.6, so $E$ lies in a maximal torus of $G$, contrary to hypothesis. Thus $r$ is 2,3 or 5 according as $G$ is $G_{2}, F_{4}$ or $E_{8}$ (by the hypothesis of the lemma). From Lemma 2.6 we see that $C_{G}(e)=X_{1} X_{2}$, a commuting product with $X_{1} \cong X_{2}$ of type $A_{1}, A_{2}$ or $A_{4}$, respectively. Moreover $Z\left(X_{1} X_{2}\right)=\langle e\rangle$.

Let $x \in X_{i} \backslash\langle e\rangle$ for some $i$, with $x$ of order $r$ (this is not possible if $G=G_{2}$ as then $X_{i} \cong \mathrm{SL}_{2}$ ). If $G=F_{4}$ then $x$ is contained in a fundamental subgroup $\mathrm{SL}_{2}$, and if $G=E_{8}$ then $x$ lies in a product of two commuting fundamental subgroups $\mathrm{SL}_{2}$. Consequently $C_{G}(x)$ contains $C_{3}, D_{6}$ in the $F_{4}, E_{8}$ cases, respectively, and we deduce that $x$ is not $G$-conjugate to $e$. Hence in any case, we may by Lemma 2.4(i) choose $e^{\prime} \in E \backslash\langle e\rangle$ with $e^{\prime}=a_{1} a_{2}$ and $a_{i} \in X_{i} \backslash\langle e\rangle$ for $i=1,2$. Note that $a_{1}$ has distinct eigenvalues on the natural module for $X_{1}$ : for otherwise $C\left(e, e^{\prime}\right)$ has a central torus, whence $C(E)$ has a normal torus, contrary to Lemma 2.5 .

By Lemma 2.4(iii) there exists $e^{\prime \prime} \in E \backslash\left\langle e, e^{\prime}\right\rangle$, and by Lemma 2.4(i) we may take $e^{\prime \prime}=b_{1} b_{2}$ with $b_{i} \in X_{i} \backslash\langle e\rangle$. We have $\left[a_{1}, b_{1}\right] \neq 1$. A straightforward calculation in $X_{1} X_{2}$ shows that $\left\langle e, e^{\prime}, e^{\prime \prime}\right\rangle$ is self-centralizing in $C_{G}(e)$, so

$$
E=C_{G}(E)=\left\langle e, e^{\prime}, e^{\prime \prime}\right\rangle \text {. }
$$

Now the group $F=\left\langle a_{1}, a_{2}, b_{1}, b_{2}\right\rangle$ is extraspecial of order $r^{5}$ and normalizes $E$, inducing a group of automorphisms of $E$ of order $r^{2}$ and centralizing $E /\langle e\rangle$ (this is a group of transvections in $\mathrm{SL}_{3}(r)$ ). Considering the above configuration, we see that beginning with the group $C_{G}\left(e^{\prime}\right) \cong C_{G}(e)$ yields a group normalizing $E$ and inducing a group of order $r^{2}$ centralizing $E /\left\langle e^{\prime}\right\rangle$. We conclude that

$$
N_{G}(E) / E \geqslant \operatorname{SL}_{3}(r) \text {. }
$$

Since $C_{G}(e) \cap C_{G}\left(e^{\prime}\right)=T\left\langle e^{\prime \prime}\right\rangle$ with $T$ a maximal torus of $X_{1} X_{2}$, it follows that no element of $N_{G}(E)$ can centralize a hyperplane of $E$ without centralizing $E$. Therefore $N_{G}(E) / E=\mathrm{SL}_{3}(r)$. This proves (i) and (ii). To complete the proof of the lemma, we must analyse the situation in the finite group $L=G_{\sigma}$.

In the case where $L={ }^{2} G_{2}(q)^{\prime}$ we have $N_{L}(E) / E \cong Z_{7}$ (see [31]), and $q=3$ by the maximality of $M$, as in Table 1 . And if $L={ }^{2} F_{4}(q)^{\prime}$ then the 3-rank of $L$ is only 2 (see $[16,10.2]$ ), so $E \$ L$, a contradiction.

Now let $L$ be $G_{2}(q)$ or $F_{4}(q)$, or $E_{8}(q)$ with $5 \mid p^{2}-1$. The subgroup $E$ lies in the $\sigma$-stable group $Y=X_{1} X_{2}$, and $O^{p^{\prime}}\left(Y_{\sigma}\right)=\operatorname{SL}_{r}^{\varepsilon}(q) \circ \operatorname{SL}_{r}^{\varepsilon}(q)$. Write $Z=\langle e\rangle$, so that $Y=C_{G}(Z)$. If $E \leqslant O^{p^{\prime}}\left(Y_{\sigma}\right)$ then the above argument shows that $E$ is conjugate to a subgroup $E_{1}$ in $\operatorname{SL}_{r}^{\varepsilon}(p) \circ \operatorname{SL}_{r}^{\varepsilon}(p)$, where $r \mid p-\varepsilon$, and $N_{G}\left(E_{1}\right)$ lies in $G_{2}(p), F_{4}(p)$ or $E_{8}(p)$ in the respective cases. Hence $q=p$ by the maximality of $M$, and $E$ is determined up to $L$-conjugacy here. Also when $L=F_{4}(2)$ we have $N(E)<L_{4}(3)<L$ (see [11, p. 170] or [25]), so this case is excluded from Table 1. Now suppose that $E \neq O^{p^{\prime}}\left(Y_{\sigma}\right)$. We may take it that $a_{1} a_{2} \in O^{p^{\prime}}\left(Y_{\sigma}\right), b_{1} b_{2} \notin$ $O^{p^{\prime}}\left(Y_{\sigma}\right)$. Now $M$ induces an irreducible subgroup of $\mathrm{SL}_{3}(r)$ on $E$ containing a 
transvection (one exists in $N_{Y_{o}}(E)$ ). Hence $M$ induces $\mathrm{SL}_{3}(r)$ on $E$ (see [24]). Consequently $N_{M}(Z)$ is transitive on $(E / Z)^{\#}$. But this is impossible as $a_{1} a_{2} \in$ $O^{p^{\prime}}\left(C(Z)_{\sigma}\right)$ and $b_{1} b_{2} \notin O^{p^{\prime}}\left(C(Z)_{\sigma}\right)$.

Finally, let $G=E_{8}$ with $5 \nmid p^{2}-1$. If $L=E_{8}(p)$ then $C_{L}(e)$ must be $\operatorname{SU}_{5}\left(p^{2}\right)$ (see $\left[13\right.$, p. 215]); but then $E$ lies in a maximal torus of $C_{L}(e)$, which is not so. Hence $q>p$. As above we see that $E \leqslant O^{p^{\prime}}\left(Y_{\sigma}\right)$. Then $E$ is conjugate to a subgroup $E_{1}$ in $\mathrm{SU}_{5}\left(p^{2}\right) \circ \mathrm{SU}_{5}\left(p^{2}\right)$, and $N_{G}\left(E_{1}\right) \leqslant E_{8}\left(p^{2}\right)$. Consequently $q=p^{2}$ here. All parts of the lemma are now proved, apart from the non-maximality of $N_{L}(E)$ when $p=2$ and $G=E_{8}$. As stated in $\S 1$, this is due to the embedding $N_{L}(E)<L_{4}(5)<E_{8}(4)$, which is demonstrated in $\S 5$.

Lemma 2.11. Suppose that $G=E_{6}$ with $r=3$. Then $E \cong\left(Z_{3}\right)^{3}, C_{G}(E)$ is special of order $3^{6}$ with derived group $E$, and $N_{G}(E) / C_{G}(E) \cong \mathrm{SL}_{3}(3)$. Moreover, $L$ and $N_{G_{\sigma}}(E)$ are as in Table 1 , and $E$ is determined uniquely up to $G_{o}$-conjugacy.

Proof. Fix $e \in E^{\#}$. By Lemmas 2.6 and 2.9(ii), we have

$$
C:=C_{G}(e)=\left(X_{1} X_{2} X_{3}\right)\left\langle w_{3}\right\rangle,
$$

where each $X_{i} \cong \mathrm{SL}_{3}, Z\left(X_{i}\right)=\langle e\rangle$ and $\left\langle w_{3}\right\rangle$ is transitive on $\left\{X_{1}, X_{2}, X_{3}\right\}$. Moreover, $E^{\#}=e^{G} \cap E$. Set $D=X_{1} X_{2} X_{3}$. For $i=1,2,3$ let $Z\left(\hat{X}_{i}^{\prime}\right)=\left\langle\hat{e}_{i}\right\rangle$. Notation may be chosen so that $\hat{e}_{1} \hat{e}_{2} \hat{e}_{3}=1, Z(\hat{G})=\left\langle\hat{e}_{1} \hat{e}_{2}^{-1}\right\rangle$ and $e=\hat{e}_{i} Z(\hat{G})$ for $i=1,2,3$. Hence $e_{1}=e_{2}=e_{3}=e$.

We next claim that if $f$ is an element of order 3 in $X_{i} X_{j} \backslash\langle e\rangle$ for some $i \neq j$, then $f$ is not conjugate to $e$. For let $J$ be a fundamental subgroup $\mathrm{SL}_{2}$ within the third $\mathrm{SL}_{3}$. Viewing $f$ as an element of $C_{G}(J)=A_{5}$, we compute that $C_{A_{5}}(f)$ contains $\mathrm{SL}_{4}$ or $\left(\mathrm{SL}_{2}\right)^{3}$, and hence that $C_{G}(f)$ contains $\mathrm{SL}_{4}$ or $\left(\mathrm{SL}_{2}\right)^{4}$, which proves the claim. Therefore, if $f \in(E \cap D) \backslash\langle e\rangle$ then $f$ has the form $a_{1} a_{2} a_{3}$ with $a_{i} \in X_{i} \backslash\langle e\rangle$ for $i=1,2,3$.

We now show that $E \leqslant D$. For suppose that there exists $f \in E \backslash D$. It is easy to check that $C \backslash\langle e\rangle$ contains precisely one conjugacy class of subgroups of order 3 lying outside $D \backslash\langle e\rangle$. Consequently $C$ contains a unique class of elementary abelian subgroups of order 9 which contain $e$ and are not contained in $D$, and $\langle e, f\rangle$ is a representative of this class. For $i=1,2,3$ let $a_{i}$ be an element of order 3 in $X_{i} \backslash\langle e\rangle$ and let $a=a_{1} a_{2} a_{3}$. Then $\left(C_{G}(e) \cap C_{G}(a)\right)^{0}=T_{6}$, a maximal torus. The possibilities for $C_{G}(a)^{0}$ can be read off from [16, 14.1]. Viewing $e$ as an element of $C_{G}(a)$ we see that the fact that $\left(C_{G}(e) \cap C_{G}(a)\right)^{0}=T_{6}$ forces $C_{G}(a)^{0}=\left(A_{2}\right)^{3}$, and hence $a$ is conjugate to $e$. For $i=1,2$ choose $b_{i}$ of order 3 in $X_{i} \backslash\langle e\rangle$ such that $\left[a_{i}, b_{i}\right]=e$, and set $b=b_{1} b_{2}^{-1}$. Then $\langle a, b\rangle$ is elementary abelian of order 9 , but $\langle\hat{a}, \hat{b}\rangle$ is not abelian. It follows that $b \notin C_{G}(a)^{0}$, and hence by the above, $\langle a, b\rangle$ is conjugate to $\langle e, f\rangle$. But this is impossible since $\langle e, f\rangle^{\#}$ is fused, while $\langle a, b\rangle^{\#}$ is not. This contradiction shows that $E \leqslant D$.

Let $f=a_{1} a_{2} a_{3} \in E \backslash\langle e\rangle$. As $C_{G}(e, f)^{0}=T_{6}$, Lemma 2.3 implies that $\left.E\right\rangle\langle e, f\rangle$, so we may pick $g=b_{1} b_{2} b_{3} \in E \backslash\langle e, f\rangle$. By the second paragraph, $b_{i} \notin\left\langle a_{i}, e\right\rangle$ for $i=1,2,3$. Replacing $g$ by $g^{2}$ if necessary, we may assume that $\left[a_{i}, b_{i}\right]=e$ for $i=1,2,3$ (since $\left[a_{i}, b_{i}\right]=1$ implies that $b_{i} \in\left\langle a_{i}, e\right\rangle$ ). It follows that $E=\langle e, f, g\rangle$.

Let $K=\left\langle a_{1}, a_{2}, a_{3}, b_{1}, b_{2}, b_{3}\right\rangle$, an extraspecial group of order $3^{7}$ normalizing $E$. We have

$$
C_{K}(E)=\left\langle E, a_{1} a_{2}^{2}, b_{1} b_{2}^{2}\right\rangle, \quad C_{K}(E)^{\prime}=\langle e\rangle=[K, E],
$$


and $C_{G}(E)=C_{K}(E)\langle t\rangle$ where $\langle t\rangle$ is transitive on $\left\{X_{1}, X_{2}, X_{3}\right\}$. Replacing $e$ by other elements of $E^{\#}$ in the above arguments, we see that $N_{G}(E) / C_{G}(E) \cong$ $\mathrm{SL}_{3}(3)$.

We now consider the finite group $L$. Here $L=E_{6}^{\varepsilon}(q)$ with $\varepsilon= \pm 1$. Now $E$ lies in the $\sigma$-stable subgroup $D=X_{1} X_{2} X_{3}$. First suppose that $3 \mid p-\varepsilon$. We see, as in the proof of Lemma 2.10 , that $E \leqslant O^{p^{\prime}}\left(D_{\sigma}\right)$, so $E$ is conjugate to a subgroup $E_{1}$ in $\operatorname{SL}_{3}^{\varepsilon}(p) \circ \mathcal{S L}_{3}^{\varepsilon}(p) \circ \mathrm{SL}_{3}^{\varepsilon}(p)$, which lies in $E_{6}^{\varepsilon}(p)$. Moreover the above argument gives $N_{G}(E) \leqslant E_{6}^{\varepsilon}(p)$ and hence $q=p$ and $E$ is determined up to $G_{\sigma}$-conjugacy here. Finally, let $3 \mid p+\varepsilon$. Then $E$ lies in a subgroup $N\left(\mathrm{SL}_{3}\left(p^{2}\right) \circ \mathrm{SL}_{3}^{-\varepsilon}(p)\right)$ of $E_{6}^{\varepsilon}(p)$ (see $\left.[14, \mathrm{p} .50]\right)$, and we find that $E$ is self-centralizing in $E_{6}^{\varepsilon}(p)$. Then by Lemma 2.10 the normalizer of $E$ in $E_{6}^{\varepsilon}(p)$ lies in a subgroup $F_{4}(p)$. Again the $G_{\sigma}$-class of $E$ is determined, so $N_{X}(E)$ is non-maximal here, a contradiction. To conclude, note that $L \neq^{2} E_{6}(2)$ here also, since in this case $N_{L}(E)$ lies in a subgroup $\Omega_{7}(3)$ of $L$ (see $\left[\mathbf{1 1}\right.$, p. 191]). Thus $L$ and $N_{G_{\sigma}}(E)$ are as in Table 1 , and the proof is complete.

Lemma 2.12. If $r=3$ then $G$ is not $E_{7}$ or $E_{8}$.

Proof. First suppose that $G=E_{7}$. Let $e \in E^{\#}$. By Lemma 2.6, $C_{G}(e)=A_{2} A_{5}$. Write $C_{G}(e) /\langle e\rangle=\bar{A}_{2} \bar{A}_{5}$ correspondingly, and let $E_{0}$ be the projection of $E$ in $\bar{A}_{5}$. If the preimage of $E_{0}$ in $A_{5}$ is abelian then $E$ is contained in a maximal torus, which is not so. Otherwise, by 2.5 , the preimage is extraspecial and $C_{G}(E)^{0}$ is a subgroup $A_{1}$ of $A_{5}$. The centralizer of this $A_{1}$ in $G$ contains $A_{2} A_{2}$ (within $C_{G}(e)$ ), and is normalized by $M=N_{X}(E)$. As $M$ is maximal, it therefore contains $C_{G}\left(A_{1}\right)_{\sigma} \cap L$. But this group does not normalize $E$.

Thus we assume that $G=E_{8}$. Then $E \leqslant K_{1} R$, a commuting product with $K_{1} \cong \mathrm{SL}_{3}$ and $R \cong \hat{E}_{6}$. Let $\left\langle e_{1}\right\rangle=Z\left(K_{1} R\right)$. Now $R \cong K_{2} K_{3} K_{4} \cong\left(A_{2}\right)^{3}$, and we write $\left\langle e_{i}\right\rangle=Z\left(K_{i}\right)$ for $i=1,2,3,4$. Let $K=K_{1} K_{2} K_{3} K_{4}$. One checks that $N_{G}(K)$ induces $S_{4}$ on $\left\{K_{1}, \ldots, K_{4}\right\}$ and $\mathrm{GL}_{2}(3)$ on $Z(K)=\left\langle e_{1}, e_{2}\right\rangle$. We may choose notation so that the relations on $\left\langle e_{1}, e_{2}, e_{3}, e_{4}\right\rangle$ are spanned by $e_{1} e_{2} e_{3}^{-1}=$ $e_{2} e_{3} e_{4}=1$.

Suppose that $g \in E^{\#}$ with $B=C_{G}(g)$ of type $A_{8}$. Let $f \in E \backslash\langle g\rangle$. Now $C_{B}(f)^{0}$ contains a normal torus, so Lemma 2.5 implies that there exists $h \in$ $E \cap\left(C_{B}(f) \backslash C_{B}(f)^{0}\right)$. It follows that $C_{B}(f)^{\circ}=\left(A_{2}\right)^{3}$. Hence $O_{3}(Z(C(E)))$ contains conjugates of $e_{1}$, and by Lemma 2.4(i) we may (and do) replace $E$ by
$\left\langle e_{1}^{G \cap} \cap O_{3}(Z(C(E)))\right\rangle$. Hence we may assume that $e_{1} \in E$.

Thus $E \leqslant C_{G}(E) \leqslant C_{G}\left(e_{1}\right)=K_{1} R$, and without loss of generality, we may assume that $E \leqslant K\langle t\rangle$, where $\langle t\rangle$ centralizes $K_{1}$ and permutes $\left\{K_{2}, K_{3}, K_{4}\right\}$
transitively. By Lemma $2.3, E$ does not transitively. By Lemma $2.3, E$ does not centralize $K_{1}$. Hence there is an element
$f=a_{1} x \in e_{1}^{G} \cap E$ with $a_{1} \in K_{1} \backslash\left\langle e_{1}\right\rangle, x \in K_{2} K_{3} K_{4}\langle t\rangle$. $f=a_{1} x \in e_{1}^{G} \cap E$ with $a_{1} \in K_{1} \backslash\left\langle e_{1}\right\rangle, x \in K_{2} K_{3} K_{4}\langle t\rangle$. By Lemma 2.3 also, there
exists $g=b_{1} y \in E$ with $\left[a_{1}, b_{1}\right]=e_{1}, b_{1} \in K_{1}$ and $y \in K_{2} K_{3} K_{4}\langle t\rangle$.
Let $b \in K_{i}$ with $|b|=9$ and $\left.b^{3} \in Z Z K_{3}\right)$ Then

Let $b \in K_{j}$ with $|b|=9$ and $b^{3} \in Z\left(K_{j}\right)$. Then a direct check shows that $b$ is not
$K_{j}$-conjugate to $b e_{j}^{ \pm 1}$. In particular, the relation $\left[a_{1}, b_{1}\right]=e_{1}$ imples thet $K_{\text {, }}$-conjugate to $b e_{j}^{ \pm 1}$. In particular, the relation $\left[a_{1}, b_{1}\right]=e_{1}$ implies that the
group $\left\langle a_{1}, b_{1}\right\rangle$ has exponent 3 .

Now $R$ is the simply connected group $\hat{E}_{6}$, which has three classes of centralizers
of non-central elements of of non-central elements of order 3 , of types $\left(A_{2}\right)^{3}, T_{2} D_{4}$ and $T_{1} A_{5}$ (since the action of $W \cong \mathrm{SO}_{5}(3)$ on $\Omega_{3}(T)$, where $T$ is a maximally split torus, has three
orbits on 1-spaces). Thus $C_{R}(x)$ is of one of these types. If $C_{R}(x)=\left(A_{2}\right)^{3}$ $f=a_{1} x$ is conjugate by an element of $R$ to $a_{1} e_{2}^{\varepsilon}$, where $\varepsilon=C_{R}(x)=\left(A_{2}\right)^{3}$ then $f=a_{1} x$ is conjugate by an element of $R$ to $a_{1} e_{2}^{\varepsilon}$, where $\varepsilon= \pm 1$. Now $N_{G}(K)$ is 
3-transitive on $\left\{K_{1}, \ldots, K_{4}\right\}$, so $K_{1} K_{2} K_{3}$ is contained in a subgroup $A_{8}$, and working within this $A_{8}$ we see clearly that $C_{G}\left(a_{1} e_{2}^{\varepsilon}\right)$ contains a subgroup $\left(A_{3}\right)^{2}$; consequently, by Lemma $2.6, C_{G}(f)$ must be $A_{8}$, contradicting the fact that $f$ is conjugate to $e_{1}$. Thus $C_{R}(x)$ is not $\left(A_{2}\right)^{3}$. If $C_{R}(x)$ is $T_{1} A_{5}$ then $x\left\langle e_{1}\right\rangle /\left\langle e_{1}\right\rangle$ is contained in a fundamental $\mathrm{SL}_{2}$ of $R /\left\langle e_{1}\right\rangle$, so its centralizer in $R /\left\langle e_{1}\right\rangle$ is connected and $C_{G}\left(e_{1}, f\right)$ contains a central torus, contrary to Lemma 2.5. Hence $C_{R}(x)$ must be $T_{2} D_{4}$.

Now at least one of the elements $f, g, f g$ and $f g^{-1}$ lies in $K$. Let $h$ be such an element, with $h=k_{1} k_{2} k_{3} k_{4}$ and $k_{i} \in K_{i}$. If $E \cap K=\left\langle e_{1}, h\right\rangle$ then $E=\left\langle e_{1}, h, e^{\prime}\right\rangle$, where either $e^{\prime}=1$ or $e^{\prime} \in E \backslash K$. In either case $C(E)$ contains a normal torus, contrary to Lemma 2.5. Therefore $\left\langle e_{1}, h\right\rangle<E \cap K$ and so we may rechoose $h$ if necessary so that $\left|k_{i}\right|=3$ for some $i>1$. Previous remarks (in the fifth paragraph of this proof) imply that $\left|k_{1}\right|=3$, and so from the relations on the elements $e_{j}$ we conclude that $\left|k_{j}\right| \leqslant 3$ for each $j$. Set $r=k_{2} k_{3} k_{4}$. We may write $h=k_{1} k_{i} k_{j}$, as otherwise $C_{R}(r)=\left(A_{2}\right)^{3}$, contradicting the previous paragraph. As $E \leqslant C(h)$ we conclude that $E \leqslant K$. We may thus take $x=a_{2} a_{3}$ with $a_{i} \in K_{i}$ and $a_{i}$ of order 3. If $K_{4} \leqslant C(E)$ then $\left(C_{G}(E)^{0}\right)^{\prime}=K_{4}$, contradicting Lemma 2.3. Hence there exists $d \in e_{1}^{G} \cap E$ such that $d=d^{\prime} d_{4}$, where $d^{\prime} \in K_{1} K_{2} K_{3}$ and $d_{4} \in K_{4}$. Then $d=d_{i} d_{j} d_{4}$ where $i \neq j, i, j \in\{1,2,3\}$ and $d_{i} \in K_{i}, d_{j} \in K_{j}$. If $\left[a_{i}, d_{i}\right] \neq 1$ or $\left[a_{j}, d_{j}\right] \neq 1$ then $[f, d] \neq 1$ since $\left\langle e_{i}, e_{j}\right\rangle \cong\left(Z_{3}\right)^{2}$. Thus $d_{i} \in\left\langle a_{i}, e_{i}\right\rangle \backslash\left\langle e_{i}\right\rangle$ and $d_{j} \in\left\langle a_{j}, e_{j}\right\rangle \backslash\left\langle e_{j}\right\rangle$.

Since $[x, y]=e_{1}^{-1}$, we have $y=b_{2} b_{3} b_{4}$ with $b_{i} \in K_{i}$ and $\left[b_{2}, a_{2}\right] \in\left\langle e_{2}\right\rangle^{\#}$, $\left[b_{3}, a_{3}\right] \in\left\langle e_{3}\right\rangle^{\#}$. But then $[g, d] \neq 1$ unless $\left[b_{4}, d_{4}\right] \in\left\langle e_{4}\right\rangle^{\#}$. Now earlier remarks imply that $\left|b_{i}\right|=3$ for all $i$. We have $g=b_{1} b_{2} b_{3} b_{4}$ with each $b_{i}$ of order 3 in $K_{i} \backslash\left\langle e_{i}\right\rangle$. But then $C_{R}\left(b_{2} b_{3} b_{4}\right)=\left(A_{2}\right)^{3}$, whereas we have seen that this centralizer should be $T_{2} D_{4}$.

This completes the proof of the lemma.

LEMMA 2.13. If $r=2$ then $G$ is not $F_{4}$ or $E_{6}$.

Proof. Suppose that the lemma is false. First let $G=E_{6}$. Let $z$ and $e$ be representatives of the two classes of involutions in $G$, with $C_{G}(z)=T_{1} D_{5}$ and $C_{G}(e)=A_{1} A_{5}$. By Lemma 2.6 we have $E^{\#}=e^{G} \cap E$, and, in particular, we may assume that $e \in E$. Write $C_{G}(e)=X D$ with $X=A_{1}, D=A_{5}$. If $d$ is an involution in $(D \cap E) \backslash\langle e\rangle$, then $C_{D}(d)=A_{1} \times A_{3}$, and setting $c=d$ or de, we have $c \in Z\left(A_{3}\right)$. Now consideration of $C_{G}(c)$ yields $c \in z^{G}$, a contradiction.

Thus $E \cap D=\langle e\rangle$, and it follows that $E$ has rank at most 3. Consequently, by Lemma $2.4, E$ has rank 3. Write $E=\langle e, f, g\rangle$. Then $C_{G}(e, f) \cap X=T_{1}$, a 1-dimensional torus inverted by $g$. As $E$ projects to a quaternion subgroup of $D$ acting homogeneously on the usual 6-dimensional module, $\left(C_{G}(E)^{0}\right)^{\prime}=K \cong A_{2}$. Lemma 2.3 implies that $C_{G}(K)^{0}$ is semisimple, and as $E$ does not centralize $T_{1}$, it cannot centralize $C_{G}(K)^{0}$. Hence by Lemma 2.1, $q=3$ and $\left(C_{G}(K)^{0}\right)_{\sigma}$ has factors $L_{2}(3)$. But $E$ has rank 3, so this contradicts Lemma 2.1(ii).

Now suppose $G=F_{4}$. Again $G$ has two classes of involutions, with representatives $e$ and $z$ such that $C_{G}(e)=A_{1} C_{3}$ and $C_{G}(z)=B_{4}$. If $E^{\#}=e^{G} \cap E$ then we obtain a contradiction as above-the only change is that here $K$ is $A_{1}$ rather than $A_{2}$.

So assume that $z \in E=\left\langle z^{G} \cap E\right\rangle$. Let $y \in\left(z^{G} \cap E\right) \backslash\langle z\rangle$. Then $C_{G}(y, z)$ is $D_{4}$ or $A_{1} A_{1} B_{2}$. In the latter case $Z\left(C_{G}(y, z)\right)=Z\left(A_{1} \times A_{1}\right)$ contains only one conjugate of $z$, a contradiction. Thus $C_{G}(y, z)=D_{4}$ and $y$ has eight eigenvalues 
-1 on the usual 9-dimensional module for $B_{4}$. The product of two commuting involutions of this type cannot again be of this type, so it follows that $E$ has rank at most 2. This contradicts Lemma 2.4 .

It remains to deal with the cases where $r=2$ and $G$ is $E_{7}$ or $E_{8}$. These require considerably more work than the previous cases.

Lemma 2.14. Suppose that $p$ is odd, $G=E_{8}$, and that $J_{1} \ldots J_{8}$ is a maximal commuting product of fundamental subgroups $\mathrm{SL}_{2}$ in $G$. Let $Z\left(J_{i}\right)=\left\langle e_{i}\right\rangle$ for $1 \leqslant i \leqslant 8$. Then with suitable ordering,

(i) $e_{1} \ldots e_{8}=1$,

(ii) $\left\langle e_{1}, \ldots, e_{8}\right\rangle \cong\left(\left\langle e_{1}\right\rangle \times \ldots \times\left\langle e_{8}\right\rangle\right) / R$, where $R=\left\langle e_{1} e_{2} e_{3} e_{8}, e_{1} e_{4} e_{5} e_{8}, e_{2} e_{4} e_{6} e_{8}, e_{3} e_{4} e_{7} e_{8}\right\rangle$,

(iii) $\left\langle e_{1}, \ldots, e_{8}\right\rangle=\left\langle e_{1}\right\rangle \times\left\langle e_{2}\right\rangle \times\left\langle e_{7}\right\rangle \times\left\langle e_{8}\right\rangle$.

Proof. Choose a subsystem $\left(A_{1}\right)^{8}$ in the $E_{8}$ root system. The elements $e_{i}$ are the elements $h_{\alpha}(-1)$ as described in [9, Chapter 6] for the roots $\alpha$ in the subsystem. A direct check using these elements gives the assertions.

Lemma 2.15. Let $r=2$ and $G=E_{7}$. Then $E=\left(Z_{2}\right)^{2}, C_{G}(E)=E \times D_{4}\left(D_{4}\right.$ of adjoint type), and $N_{G}(E) / C_{G}(E) \cong S_{3}$. Moreover, $L$ and $N_{G_{\sigma}}(E)$ are as in Table 1, and $E$ is determined up to $G_{\sigma}$-conjugacy.

Proof. First note that $G$ has precisely three classes of involutions, with centralizers $A_{1} D_{6}, A_{7}\left\langle w_{2}\right\rangle$ and $T_{1} E_{6}\left\langle w_{2}\right\rangle$. Of these, the first lifts to a class of involutions in $\hat{E}_{7}$, while the other two lift to elements of order 4.

Let $\langle z\rangle=Z(\hat{G})$. By Lemma 2.9(i), $\hat{E}$ is either extraspecial or elementary abelian. Suppose that $\hat{E}$ is extraspecial. Then $\hat{e}$ is conjugate to $\hat{e} z$ for every $\hat{e} \in \hat{E}$, and hence $C_{G}(e)^{0}<C_{G}(e)$ for each $e \in E^{\#}$. Hence Lemmas 2.6 and 2.9(ii) imply that each element of $E^{\#}$ has centralizer $A_{7}\left\langle w_{2}\right\rangle$. Consequently, every element of $\hat{E} \backslash\langle z\rangle$ has order 4 , and so $\hat{E} \cong Q_{8}$ and $\hat{E}=\langle\hat{e}, \hat{f}\rangle$ with $[\hat{e}, \hat{f}]=z$.

Let $y$ be an involution of $G$ with centralizer $T_{1} E_{6}\left\langle w_{2}\right\rangle$ and let $d$ be an involution in $C_{G}(y) \backslash C_{G}(y)^{0}$. (To see that $d$ exists, let $T$ be a maximal torus of $T_{1} E_{6}$ and let $T \leqslant J_{1} \ldots J_{7}$, a maximal commuting product of fundamental subgroups $\mathrm{SL}_{2}$. Let $s_{i}$ be a fundamental reflection in $J_{i}$ and set $d=s_{1} \ldots s_{7}$. Then $\alpha^{d}=-\alpha$ for each root $\alpha$ and so $d$ induces a graph automorphism on the factor $E_{6}$. Finally, $d$ is an involution.) Write $C=\left(C_{G}(y)^{0}\right)^{\prime} \cong E_{6}$. The proof of Proposition 2.7 shows that there is an involution $h$ in a fundamental subgroup $\mathrm{SL}_{2}$ of $K=C \cap C_{G}(d)$ such that $d, d h$ are representatives of the two classes of involutions in $C d$. Suppose that $d, d h \in y^{G}$. Then

$$
C_{G}(d, d h)^{0}=\left(C_{G}(d)^{0} \cap C_{G}(h)\right)^{0}=T_{1} A_{1} A_{5} .
$$

Hence $d^{g}=d h$ implies that $g$ normalizes $T_{1}$. Then $g$ normalizes $C_{G}\left(T_{1}\right)=C_{G}(y)^{0}$. iut $d, d h$ are not conjugate in $N_{G}\left(C_{G}(y)^{0}\right)=C_{G}(y)$, a contradiction. Thus $d, d h$ ee not both conjugates of $y$. Now $d \in C(y) \backslash C(y)^{0}$, so $[\hat{d}, \hat{y}] \neq 1$ and therefore ${ }_{5}(d)^{0}<C_{G}(d)$. Similarly $C_{G}(d h)^{0}<C_{G}(d h)$. It follows that at least one of $d, d h$ conjugate to an element of $E^{\#}$. Consequently, we may choose $e \in E^{\#}$ such that $y$ commute but $\hat{e}, \hat{y}$ do not commute. 
We now have the two non-conjugate involutions $f, y \in C(e) \backslash C(e)^{0}$. Moreover, since $e \in C(y) \backslash C(y)^{0}$, Proposition 2.7 implies that $\left(C(e) \cap C(y)^{0}\right)^{\prime}$ is of type $F_{4}$ or $C_{4}$; as this subgroup lies in $C(e)^{0}=A_{7}$, it must be of type $C_{4}$. Hence, also by Proposition 2.7, $C(e)^{0} \cap C(f)^{0}=D_{4}$, that is, $C_{G}(E)^{0}=D_{4}$ (of adjoint type). Further, $E^{\#}=e^{G} \cap E$ and $f$, $f e$ are conjugate in $C_{G}(e)=A_{7}\left\langle w_{2}\right\rangle$ (regarding the action of $f$ as the 'inverse-transpose' action, $f$ and $f e$ are conjugate by a suitable diagonal element of $A_{7}$ ). Repeating this for each element of $E^{\#}$, we see that $N_{G}(E) / C_{G}(E) \cong S_{3}$. We claim that the group $S_{3}$ here acts as graph automorphisms on the factor $D=C_{G}(E)^{0} \cong D_{4}$. For suppose to the contrary that there is a 3-element $x$ centralizing $D$. Now $D$ has two composition factors on the minimal 56-dimensional module for $G$, each of dimension 28 (each is the skew-square of a natural 8-dimensional module). It follows that $x$ has precisely two distinct eigenvalues on the module, and $C_{G}(x)$ acts on the corresponding eigenspaces, each of which has dimension 28. But $D$ is irreducible on each eigenspace, so by $[16,14.1]$ the only possibility for $C_{G}(x)^{0}$ is $A_{7}$. This is absurd as $A_{7}$ centralizes only 2-elements. Hence our claim is proved.

To conclude this case (assuming $\hat{E}$ extraspecial), we consider the situation in the finite group $G_{\sigma}$. We have proved that the $G$-class of $E$ is uniquely determined, and $N_{G}(E)=\left(E \times D_{4}\right) . S_{3}$. Applying [26, I, 2.7 and 2.8], we see that the $G_{\sigma}$-classes of subgroups $\left(E_{1}\right)_{\sigma}$ for $\sigma$-stable $E_{1} \in E^{G}$ are in bijective correspondence with the classes of elements in the coset $S_{4} \sigma=\left(E . S_{3}\right) \sigma$. The only such classes giving Klein 4-groups $\left(E_{1}\right)_{\sigma}$ are represented by $\sigma$ and $e \sigma$, where $e \in E^{\#}$. In the latter case, however, the corresponding Klein 4-group does not have an element of order 3 acting on it. Hence the $G_{\sigma}$-class of $E$ is uniquely determined, and $N_{G_{o}}(E)=\left(E \times \operatorname{Inndiag}\left(D_{4}(q)\right) \cdot S_{3}\right.$, as in Table 1 .

We may now assume that $\hat{E}$ is elementary abelian. Thus each involution in $E^{\#}$ has centralizer of type $A_{1} D_{6}$. We view $\hat{G}$ as a subgroup of $E_{8}$, centralizing a fundamental subgroup $\mathrm{SL}_{2}$, say $J_{8}$ (in the notation of Lemma 2.14). We may then use the relations in Lemma 2.14, setting $\langle z\rangle=\left\langle e_{8}\right\rangle=Z(\hat{G})$, and $\hat{e}=e_{1}$. Let $C_{\hat{G}}(\hat{e})=J_{1} Y$, a commuting product of $J_{1} \cong A_{1}$ and $Y \cong D_{6}$. Note that $e_{1}$ and $e_{1} z$ are not conjugate in $\hat{G}$, since otherwise $C_{G}(e)$ would not be connected, a contradiction.

Suppose that there exists $\hat{f} \in(\hat{E} \cap Y) \backslash\langle\hat{e}, z\rangle$. Choose a maximal torus $T$ of $J_{1} Y$ containing $\hat{e}$ and $\hat{f}$. Then $J_{1}$ is contained in $J_{1} T \leqslant C_{\hat{G}}(\hat{f})=J_{1}^{g} Y^{g}$ for some $g \in G$. Since $J_{1}$ is $T$-invariant, it follows that $J_{1} \leqslant Y^{g}$ and so $\left[J_{1}, J_{1}^{g}\right]=1$. Hence $\hat{f} \in J_{1}^{g} \leqslant C\left(J_{1}\right)=Y$. Thus $\hat{f}$ lies in a fundamental $\mathrm{SL}_{2}$ of $Y$, so

$$
\hat{E} \leqslant C_{G}(\hat{e}, \hat{f})=C_{\hat{G}}(\hat{e}, \hat{f})^{0}=J_{1} J_{2} J_{3} R \text {, }
$$

where $J_{1}, J_{2}, J_{3}$ are conjugate fundamental subgroups $\mathrm{SL}_{2}$, and $R \cong D_{4}$. Letting $\left\langle e_{i}\right\rangle=Z\left(J_{i}\right)$, we have $Z\left(J_{1} J_{2} J_{3} R\right)=\left\langle e_{1}\right\rangle \times\left\langle e_{2}\right\rangle \times\left\langle e_{3}\right\rangle$ and $Z\left(J_{1} J_{2} J_{3}\right) \cap Z(R)=$ $\left\langle e_{1} e_{2}, e_{2} e_{3}\right\rangle$. By Lemma 2.3, $C_{J_{i}}(E)^{0}=1$ for $i=1,2,3$, so there exist elements $a_{1} a_{2} r_{1}, b_{1} b_{2} r_{2}, a_{2}^{\prime} a_{3} r_{3}, b_{2}^{\prime} b_{3} r_{4} \in \hat{E}$ such that $a_{i}$ and $b_{i}$ (also $a_{2}^{\prime}$ and $b_{2}^{\prime}$ ) are elements of order 4 in $J_{i}$ with $\left[a_{i}, b_{i}\right]=e_{i}$ (also $\left[a_{2}^{\prime}, b_{2}^{\prime}\right]=e_{2}$ ), and $r_{1}, r_{2}, r_{3}, r_{4} \in R$. But then either $\left[a_{2}, a_{2}^{\prime}\right] \neq 1$ or $\left[a_{2}, b_{2}^{\prime}\right] \neq 1$, say the latter. This forces $\left[a_{1} a_{2} r_{1}, b_{2}^{\prime} b_{3} r_{4}\right] \neq 1$, a contradiction. Thus there is no such element $\hat{f}$.

By Lemmas 2.3 and 2.5 , there exist $x_{1}=a_{1} y_{1}, x_{2}=b_{1} y_{2} \in \hat{E}$ with $a_{1}, b_{1} \in J_{1}$, $y_{1}, y_{2} \in Y$ and $a_{1}^{2}=b_{1}^{2}=y_{1}^{2}=y_{2}^{2}=\left[a_{1}, b_{1}\right]=\left[y_{1}, y_{2}\right]=e_{1}$. The previous paragraph implies that

$$
\hat{E}=\left\langle e_{1}, z, x_{1}, x_{2}\right\rangle
$$


Now $Y /\langle z\rangle$ is a half-spin group: to see this, regard $Y /\langle z\rangle$ as a Levi factor of $G$; the corresponding unipotent radical is a 32-dimensional spin module for a half-spin group of type $D_{6}$. Since $z$ is conjugate to $e_{1}$ in $N_{E_{8}}(Y), Y /\left\langle e_{1}\right\rangle$ is also a half-spin group, and hence $Y /\left\langle z e_{1}\right\rangle \cong \mathrm{SO}_{12}$. Moreover, $y_{1}\left\langle z e_{1}\right\rangle$ and $y_{2}\left\langle z e_{1}\right\rangle$ are elements of order 4 squaring to the central involution. Consequently

$$
C_{\hat{G}}\left(\hat{e}, z, x_{1}\right)^{n}=T_{2} A_{5} \text {. }
$$

Further, $x_{2}=b_{1} y_{2}$ inverts $T_{2}$ and acts as a graph automorphism on $A_{5}$. Thus by Proposition $2.7, K=C_{G}(E)^{0}$ is of type $C_{3}$ or $D_{3}$. Also $T_{2} \leqslant C(K)^{0}$. Since $x_{2}$ inverts $T_{2}, E$ does not centralize $C(K)^{0}$, so by Lemma $2.1, q=3$ and $\left(C(K)^{(1)}\right)_{\sigma}$ has factors $L_{2}(3)$. But the rank of $E$ is 3 , which contradicts Lemma 2.1(ii).

For the final lemma, handling the case where $G=E_{8}$ and $r=2$, we require the following elementary proposition.

PROPOSITION 2.16. Let $D$ be an elementary abelian group of order $2^{\alpha} \geqslant 4$, and let $V$ be a module for $D$ over a field of odd characteristic. Then

$$
V=C_{V}(D) \oplus \sum C_{V}^{0}\left(D_{0}\right)
$$

where the sum is over the subgroups $D_{0}$ of index 2 in $D$, and $C_{V}^{(0}\left(D_{0}\right)$ is the unique '-invariant complement to $C_{V}(D)$ in $C_{V}\left(D_{0}\right)$. In particular, if $f=\operatorname{dim} C_{V}(D)$ then

$$
\operatorname{dim} V+\left(2^{\alpha}-2\right) f=\sum \operatorname{dim} C_{V}\left(D_{0}\right) .
$$

Proof. This follows from $[\mathbf{1 5}, 3.3 .3]$.

Lemma 2.17. Suppose that $G=E_{8}$ and $r=2$. Then $E=\left(Z_{2}\right)^{5}, C_{G}(E) / E \cong$ $\left(Z_{2}\right)^{10}, \quad C_{G}(E)^{\prime}=E$ and $N_{G}(E) / C_{G}(E) \cong \mathrm{SL}_{5}(2)$. Moreover, $L=E_{8}(p)$ and $N_{L}(E)=N_{G}(E)$ as in Table 1 , and $E$ is uniquely determined up to L-conjugacy.

Proof. Let $e, z$ be involutions in $G$ with $C_{G}(z)=D \cong D_{8}$ and $C_{G}(e)=J_{1} Y$, where $J_{1}$ is a fundamental subgroup $\mathrm{SL}_{2}$ and $Y$ is simply connected of type $E_{7}$. Note that $D$ is a half-spin group (see [17]).

Let $F=J_{1} \ldots J_{8}$ be as in Lemma 2.14 , with $\left\langle e_{i}\right\rangle=Z\left(J_{i}\right)$ for $1 \leqslant i \leqslant 8$. Take $F \leqslant D$, so that $z \in Z(F)$. The group $N_{G}(F) / F$ induces $2^{3} . \mathrm{SL}_{3}(2)$ on $F$ (see [2]), and $Z(F)=\left\langle e_{1}, e_{2}, e_{7}, e_{8}\right\rangle$ has two $N_{G}(F)$-classes of involutions: $e^{G} \cap Z(F)=$ $\left\{e_{i} \mid 1 \leqslant i \leqslant 8\right\}$, and $z^{G} \cap Z(F)=\left\{e_{i} e_{j} \mid i \neq j\right\}$. Take $e=e_{1}$ and $z=e_{1} e_{8}$.

Throughout, $a_{i}$ and $b_{i}$ denote elements of order 4 in $J_{i}$ satisfying $a_{i}^{2}=b_{i}^{2}=$ $\left[a_{i}, b_{i}\right]=e_{i}$. We divide the proof into steps.

Step 1. Involutions in $D$. We make some observations concerning involutions in $D$. If $x$ is an involution in $D$ such that $C_{D}(x)=S_{1} S_{2}$ with $S_{1}$ and $S_{2}$ of type $D_{4}$, then $Z\left(C_{G}(z, x)\right)=\langle z, x\rangle$ and $z, x, x z$ are all conjugate. We may take $J_{1} J_{2} J_{3} J_{8} \leqslant$ $S_{1}$ and $J_{4} J_{5} J_{6} J_{7} \leqslant S_{2}$. Then $Z\left(S_{1}\right)=Z\left(S_{2}\right)=\left\langle e_{1} e_{2}, e_{2} e_{3}\right\rangle=\left\langle e_{4} e_{7}, e_{4} e_{5}\right\rangle$.

Next, $D$ contains two classes of subgroups $A_{7}$. In one class the groups $A_{7}$ are isomorphic to $\mathrm{SL}_{8}$, with central involution $z$. The groups $A_{7}$ in the other class have centre of order 4 and do not contain $z$; the central involutions in these latter groups $A_{7}$ are conjugates of $e$. Note that a subgroup $A_{7}$ of $\hat{E}_{7}$ has centre of order 4 . 
Step 2. Involutions in $F$. Let $\Delta=\left\{\{i, j, k, l\} \mid e_{i} e_{j} e_{k} e_{l}=1\right\}$. The 4-sets in $\Delta$ are determined by Lemma 2.14(ii). We have $|\Delta|=14$ and $N_{G}(F) / F=\mathrm{AGL}_{3}(2)$ is transitive on $\Delta$. Each 4-set in $\Delta$ corresponds to a product of two groups $\mathrm{SO}_{4}$ in an $N(F)$-conjugate of $D$. For an involution $t \in D$ corresponding to an element of $\mathrm{SO}_{16}$ with $a$ eigenvalues -1 and $b$ eigenvalues +1 (with $a+b=16$ ), we say that $t$ is of type $(-1)^{a}(1)^{b}$. Thus

$$
\begin{aligned}
& e=e_{1} \text { is of type }(-1)^{4}(1)^{12}, \\
& e_{1} e_{8}=z \text { is of type }(-1)^{8}(1)^{8} .
\end{aligned}
$$

The involutions in $F \backslash Z(F)$ are $a_{1} \ldots a_{8}$ and $a_{i} a_{j} a_{k} a_{l} e_{r}^{s}$, where $\{i, j, k, l\} \in \Delta$ and $r \notin\{i, j, k, l\}, s=0$ or 1 . Calculation in $D$ gives:

$$
\begin{aligned}
& a_{1} \ldots a_{8} \text { is of type }(-1)^{8}(1)^{8}, \text { conjugate to } e_{1} e_{8}, \\
& a_{i} a_{j} a_{k} a_{l} \text { is of type }(-1)^{4}(1)^{12} \text {, conjugate to } e_{1}, \\
& a_{i} a_{j} a_{k} a_{l} e_{r}(r \notin\{i, j, k, l\}) \text { is of type }(-1)^{8}(1)^{8}, \text { conjugate to } e_{1} e_{8} .
\end{aligned}
$$

Note also that $C_{D}\left(a_{1} \ldots a_{8}\right)^{0} \cong C_{D}\left(a_{i} a_{j} a_{k} a_{l} e_{r}\right)^{0} \cong D_{4} D_{4}$.

The remainder of the proof falls into two sections: in Part $A$ we show that $E^{\#}=z^{G} \cap E$, that is, $E$ is a $z$-group; and in Part B we show that the $z$-group $E$ is the group $\left(Z_{2}\right)^{5}$ in the conclusion of the lemma.

PART A. E is a $z$-group.

Suppose that this is false, and take $e \in E$. Write $E_{0}=E \cap Y$ (recall that $C_{G}(e)=J_{1} Y$ with $\left.Y=\hat{E}_{7}\right)$.

Step 3. There exists $f \in E_{0} \backslash\langle e\rangle$. By Lemmas 2.3 and 2.5, there exist $a_{1} y$, $b_{1} y^{\prime} \in E$ such that $a_{1}, b_{1} \in J_{1}, y, y^{\prime} \in Y$ and

$$
a_{1}^{2}=b_{1}^{2}=y^{2}=\left(y^{\prime}\right)^{2}=\left[a_{1}, b_{1}\right]=\left[y, y^{\prime}\right]=e .
$$

Suppose that $E_{0}=\langle e\rangle$. Then $E=\left\langle e, a_{1} y, b_{1} y^{\prime}\right\rangle$. Now $C_{G}\left(a_{1} y\right) \cap J_{1}=T_{1}$ and $b_{1}$ inverts $T_{1}$. Moreover, $C\left(e, a_{1} y\right)^{0}$ is $T_{1} A_{7}$ or $T_{1} T_{1}^{\prime} E_{6}$ (see Lemma 2.15 for the involution classes in $Y$ ), and $y^{\prime}$ acts as a graph automorphism on $\left(C\left(e, a_{1} y\right)^{0}\right)^{\prime}$. Thus by Proposition 2.7, $\left(C(E)^{0}\right)^{\prime}=K$ is of type $F_{4}, D_{4}$ or $C_{4}$. Clearly $K$ centralizes $T_{1}$, so $T_{1} \leqslant C(K)^{0}$. Moreover $E \cap C(K)^{0}$ contains $e$, so $E \leqslant C(K)^{0}$. Since $b_{1} y^{\prime}$ inverts $T_{1}, E$ does not centralize $C(K)^{0}$. Hence Lemma 2.1(ii) applies to give a contradiction.

Step 4. The subgroup $C_{G}(e, f)$. In this step we calculate $C_{G}(e, f)$ (where $f$ is as given by Step 3). As $Y$ is simply connected, $C_{Y}(f)$ is $A_{1} D_{6}, A_{7}$ or $T_{1} E_{6}$. The last possibility does not hold, by Lemma $2.5(\mathrm{i})$; and in the second case $C_{Y}(f) \cong$ $\mathrm{SL}_{8} / Z_{2}$, forcing $f=e$, which is false. Hence $C_{G}(e, f)=J_{1} C_{Y}(f) \cong A_{1} A_{1} D_{6}$. We may take

$$
C_{G}(e, f)=J_{1} J_{8} R
$$

where $R \cong D_{6}$. Then $\langle e, f\rangle=Z\left(C_{G}(e, f)\right)=\left\langle e_{1}, e_{8}\right\rangle$, and $z \in E$. Also $J_{1} J_{8} \cap R=$ $\left\langle e_{1}, e_{8}\right\rangle$. 
Step 5. We have $E \cap R=\left\langle e_{1}, e_{8}\right\rangle$. Suppose that the claim is false, and pick $g \in(E \cap R) \backslash\left\langle e_{1}, e_{8}\right\rangle$. Then $C_{R}(g) \cong A_{1} A_{1} D_{4}$. We may take $g=e_{2}, C_{R}(g)=$ $J_{2} J_{3} S_{2}$, so that $C_{G}\left(e_{1}, e_{8}, g\right)=J_{1} J_{2} J_{3} J_{8} S_{2}$ (recall that $\left.S_{2} \cong D_{4}\right)$.

First assume that there also exists $h \in E \cap\left(S_{2} \backslash Z\left(S_{2}\right)\right)$. We may take $C_{S_{2}}(h)=$ $J_{4} J_{5} J_{6} J_{7}$, so that $E \leqslant F$ and $Z=Z(F) \leqslant E$. For $1 \leqslant i \leqslant 8$ choose $a_{i}, b_{i} \in J_{i}$ of order 4 with $\left[a_{i}, b_{i}\right]=e_{i}$. For a 4-set $A=\{\alpha, \beta, \gamma, \delta\} \in \Delta$, write

$$
a_{A}=a_{\alpha} a_{\beta} a_{\gamma} a_{\delta}, \quad b_{A}=b_{\alpha} b_{\beta} b_{\gamma} b_{\delta} .
$$

Define the subgroups $U, Y_{A}(A \in \Delta)$ of $F$ as follows:

$$
\begin{aligned}
& \left.U=\left\langle Z, a_{B}, b_{1} \ldots b_{8}\right| \text { all } B \in \Delta\right\rangle, \\
& Y_{A}=\left\langle Z, a_{A}, b_{A}, a_{\bar{A}}, b_{\bar{A}}\right\rangle,
\end{aligned}
$$

where $\bar{A}$ denotes the complement of $A$ in $\{1, \ldots, 8\}$. Thus $U \cong\left(Z_{2}\right)^{9}$ and $Y_{A} \cong\left(Z_{2}\right)^{8}$. Moreover, if $\left.U_{0}=\left\langle Z, a_{B}\right| B \in \Delta\right\}$, the coset $U_{0} b_{1} \ldots b_{8}$ in $U$ consists entirely of conjugates of $z$.

We claim now that for some $A \in \Delta$ and choice of $a_{i}, b_{i}$, the group $E$ is contained in either $U$ or $Y_{A}$. To see this, note that if $E$ contains $a_{B}$ and $a_{C}$ with $B$, $C \in \Delta$ and $|B \cap C|=2$, then $E$ can contain no element $b_{A}$ (as $E$ is abelian), and hence $E \leqslant U$. Otherwise, the set $\left\{a_{B} \mid a_{B} \in E\right\}$ is contained in $\left\{a_{A}, a_{\bar{A}}\right\}$ for some $A \in \Delta$, and then clearly $E \leqslant Y_{A}$, proving the claim.

If $E \leqslant U$ then $\left\langle e^{G} \cap E\right\rangle \leqslant U_{0}$, and hence $E \leqslant U_{0}$ by Lemma 2.4; but clearly $U_{0}$ lies in a maximal torus of $F$, so this contradicts our hypothesis on $E$. Hence $E \leqslant Y_{A}$ for some $A \in \Delta$ and choice of $a_{i}, b_{i}$. Now $E$ centralizes no $J_{i}$ by Lemma 2.3 , and hence we may assume that $a_{A} a_{\bar{A}}=a_{1} \ldots a_{8} \in E$. Further, $C_{F}\left(a_{1} \ldots a_{8}\right)^{10}=$ $T_{8}$. By Lemma $2.5, E$ centralizes no torus in $T_{8}$, and hence $E$ also contains $b_{A} b_{\bar{A}}=b_{1} \ldots b_{8}$. Thus

$$
\left\langle Z, a_{1} \ldots a_{8}, b_{1} \ldots b_{8}\right\rangle \leqslant E \leqslant Y_{A} .
$$

If $|E|=2^{6}$ then $\left\langle e^{G} \cap E\right\rangle \leqslant Z<E$, a contradiction. If $|E|=2^{7}$ then we may take $E=\left\langle Z, a_{A}, a_{\bar{A}}, b_{1} \ldots b_{8}\right\rangle$ and then $b_{1} \ldots b_{8} \notin\left\langle e^{G} \cap E\right\rangle$, again a contradiction. Thus $|E|=2^{8}$ and $E=Y_{A}$. Write $A=\{i, j, k, l\}$. One checks now that $\left\langle e_{i} e_{j}, e_{i} e_{k}\right\rangle$ is the unique largest $z$-pure subgroup of $Y_{A}$ whose involutions $t$ satisfy $t x \in z^{G} \cup\{1\}$ for all $x \in E \cap z^{G}$. Consequently $N_{G}(E)$ normalizes $\left\langle e_{i} e_{j}, e_{i} e_{k}\right\rangle$, which lies in a torus, contrary to hypothesis.

Thus there is no such element $h \in E \cap\left(S_{2} \backslash Z\left(S_{2}\right)\right)$.

We have $E \leqslant J_{1} J_{2} J_{3} J_{8} S_{2}$ and $\left\langle e_{1}, e_{2}, e_{8}\right\rangle \leqslant E$. Since $E \cap\left(S_{2} \backslash Z\left(S_{2}\right)\right)=\varnothing$, elements $z^{\prime}$ of $\left(E \cap z^{G}\right) \backslash\left\langle e_{1}, e_{2}, e_{8}\right\rangle$ have the form $a_{1} a_{2} a_{3} a_{8} s$ or $a_{i} a_{j} s$ with $s \in S_{2}$ : for if $x s \in E$ with $x \in Z\left(J_{1} J_{2} J_{3} J_{8}\right)$ then $s \in E$, a contradiction. If $z^{\prime}=a_{1} a_{2} a_{3} a_{8} s$, then $z^{\prime}\left\langle e_{1}, e_{2}, e_{8}\right\rangle$ is fused (that is, consists entirely of $z$-conjugates), and if $z^{\prime}=a_{i} a_{j} s$ then $z^{\prime}\left\langle e_{i}, e_{j}\right\rangle$ is fused.

Suppose that $E$ contains $a_{i} a_{j} s_{1}$ with $s_{1} \in S_{2}$, and let $\{i, j, k, l\}=\{1,2,3,8\}$. Then there exist $s_{2}, s_{3}, s_{4} \in S_{2}$ such that

$$
E \leqslant\left\langle e_{1}, e_{2}, e_{8}, a_{i} a_{j} s_{1}, b_{i} b_{j} s_{2}, a_{k} a_{l} s_{3}, b_{k} b_{l} s_{4}\right\rangle .
$$

It follows that the intersection of all maximal $z$-pure subgroups of $E$ must contain $e_{i} e_{j}$, and hence must be a proper non-trivial subgroup of $E$ normalized by $M$, which is a contradiction. Consequently $E$ contains no such element $a_{i} a_{j} s_{1}$, and so there exist $s_{1}, s_{2} \in S_{2}$ such that

$$
E \leqslant\left\langle e_{1}, e_{2}, e_{8}, a_{1} a_{2} a_{3} a_{8} s_{1}, b_{1} b_{2} b_{3} b_{8} s_{2}\right\rangle .
$$


Lemmas 2.3 and 2.5 force equality here, and Lemma 2.4(i) allows us to take $a_{1} a_{2} a_{3} a_{8} s_{1}, b_{1} b_{2} b_{3} b_{8} s_{2} \in z^{G}$. But then $\left\langle E \cap e^{G}\right\rangle<E$, a contradiction.

Thus the element $g$ does not exist, and so $E \cap R=\left\langle e_{1}, e_{8}\right\rangle$, completing Step 5.

From Step 5 it follows that

$$
E \leqslant\left\langle e_{1}, e_{8}, a_{8} r_{1}, b_{8} r_{2}, a_{1} r_{3}, b_{1} r_{4}\right\rangle,
$$

where $r_{i} \in R \cong D_{6}$ for $1 \leqslant i \leqslant 4$.

Step 6. We have $a_{8} r_{1} \notin E$. Suppose that $a_{8} r_{1} \in E$. Recall that $a_{8} r_{1} \in Y \cong E_{7}$, where $C_{G}\left(e_{1}\right)=J_{1} Y$. Since $Y$ is simply connected, we have $C_{Y}\left(a_{8} r_{1}\right)=A_{1} D_{6}$ (see Lemma 2.15 for the involution classes of $Y$ ), and $Z\left(D_{6}\right)=\left\langle a_{8} r_{1}, e_{1}\right\rangle$. From Step 2 we see that $a_{8} r_{1}$ and $a_{8} r_{1} e_{1}$ are not conjugate in $G$, and hence we may assume that $a_{8} r_{1} \in z^{G}$. Similarly we may take $b_{8} r_{2}, a_{1} r_{3}$ and $b_{1} r_{4}$ to lie in $z^{G}$. We now compute connected centralizers as follows. First, we have

$$
C_{G}\left(e_{8}, a_{8} r_{1}\right)^{0}=T_{1} A_{7},
$$

since $a_{8} r_{1} \in z^{G}$ and $r_{1}$ is an element of order 4 in the factor $E_{7}$ of $C_{G}\left(e_{8}\right)$. Next,

$$
C_{G}\left(e_{8}, a_{8} r_{1}, e_{1}\right)^{0}=T_{2} J_{1} A_{5},
$$

since $C\left(e_{1}, e_{8}\right)^{0}=J_{1} J_{8} R$ and $r_{1}$ is an element of order 4 in $R$. Also,

$$
C_{G}\left(e_{8}, a_{8} r_{1}, e_{1}, b_{8} r_{2}\right)^{0}=J_{1} C_{3} \text { or } J_{1} D_{3},
$$

since $r_{2}$ inverts $r_{1}$, hence interchanges the two distinct 6-dimensional eigenspaces of $r_{1}$ on the natural 12-dimensional $R$-module, and hence induces a graph automorphism on the factor $A_{5}$ in $C_{G}\left(e_{8}, a_{8} r_{1}, e_{1}\right)^{0}$ : now use Proposition 2.7. Next,

$$
C_{G}\left(e_{8}, a_{8} r_{1}, e_{1}, b_{8} r_{2}, a_{1} r_{3}\right)^{0}=T_{2} A_{2},
$$

since $r_{3}$ lies in $C_{3}$ or $D_{3}$. Finally,

$$
C_{G}\left(e_{8}, a_{8} r_{1}, e_{1}, b_{8} r_{2}, a_{1} r_{3}, b_{1} r_{4}\right)^{0}=A_{1},
$$

since $r_{4}$ inverts $r_{3}$.

Now recall that $\left\langle e_{1}, e_{8}, a_{8} r_{1}\right\rangle \leqslant E$. Let $T_{1}=Z\left(C_{G}\left(e_{8}, a_{8} r_{1}\right)^{0}\right)$. Since $C_{G}(E)$ has no normal torus by Lemma $2.5, E$ contains an element inverting $T_{1}$. From the above calculations we see that $K=C_{G}(E)^{0} \neq 1$ and $T_{1} \leqslant C_{G}(K)^{0}$. Moreover $e_{8} \in E \cap C_{G}(K)^{0}$, so $E \leqslant C_{G}(K)^{0}$. Now $E$ does not centralize $C_{G}(K)^{0}$, so Lemma 2.1(ii) applies. Thus the rank $m(E)$ of $E$ is even, so it is 4 or 6 . If $m(E)=6$ then $K=C_{G}(E)^{0} \cong A_{1}$ and $M$ normalizes $K_{\sigma} \cong \mathrm{PGL}_{2}(3)$. But then $M$ normalizes $\mathrm{O}_{2}\left(K_{\sigma}\right) \cong\left(Z_{2}\right)^{2}$, which lies in a torus of $G_{\sigma}$.

Therefore $m(E)=4$. Now $C\left(e_{1}, e_{8}, a_{8} r_{1}\right)^{0}=T_{2} J_{1} A_{5}$, so if $C=C(E)^{0} \cap A_{5}$ then $C^{\prime}$ is a group of rank at least 2 , normalized by $M$. Then $C_{G}\left(C^{\prime}\right)$ contains $T_{1}$, so does not centralize $E$, and hence Lemma 2.1(ii) applies to $C_{G}\left(C^{\prime}\right)$. This is impossible as $C_{G}\left(C^{\prime}\right)$ contains $J_{1} J_{8}$.

Thus $a_{8} r_{1} \notin E$, and Step 6 is complete.

Similarly $b_{8} r_{2}, a_{1} r_{3}, b_{1} r_{4} \notin E$. Consequently, by Lemma 2.5 we may assume that

$$
E=\left\langle e_{1}, e_{8}, a_{1} a_{8} r, b_{1} b_{8} r^{\prime}\right\rangle
$$


where $\quad r, r^{\prime} \in R \cong D_{6}$. The cosets $a_{1} a_{8} r\left\langle e_{1}, e_{8}\right\rangle, \quad b_{1} b_{8} r^{\prime}\left\langle e_{1}, e_{8}\right\rangle$ and $a_{1} a_{8} r b_{1} b_{8} r^{\prime}\left\langle e_{1}, e_{8}\right\rangle$ are each fused in $J_{1} J_{8}$. Hence it is not possible that $E=\left\langle e^{G} \cap E\right\rangle=\left\langle z^{G} \cap E\right\rangle$, contrary to Lemma 2.4.

This completes Part $\mathrm{A}$. Thus from now on we assume that $E$ is a $z$-group, and that $z=e_{1} e_{8} \in E$.

PART B. $E \cong\left(Z_{2}\right)^{5}$ is as in the conclusion of the lemma.

We begin by producing the $z$-pure subgroups $\left(Z_{2}\right)^{5}$ of the conclusion.

Step 7. Let $F=J_{1} \ldots J_{8}$ with each $J_{i}$ a fundamental subgroup $\mathrm{SL}_{2}$, and $Z\left(J_{i}\right)=\left\langle e_{i}\right\rangle$, as above. Let $Z_{0}=\left\langle e_{i} e_{j}\right|$ all $\left.i, j\right\rangle$. Define

$$
A=\left\langle Z_{0}, a_{1} \ldots a_{8}, b_{1} \ldots b_{8}\right\rangle \text {. }
$$

Then:

(i) $A$ is a $z$-group and $A \cong\left(Z_{2}\right)^{5}$; any $z$-pure subgroup $\left(Z_{2}\right)^{5}$ of $F$ containing $Z_{0}$ is $F$-conjugate to $A$;

(ii) $C_{G}(A)$ is a special group of order $2^{15}$, with $C_{G}(A)^{\prime}=A$ and $C_{G}(A) / A \cong$ $\left(Z_{2}\right)^{10}$

(iii) $X=N_{G}(A) / C_{G}(A) \cong \mathrm{SL}_{5}(2)$, and the action of $X$ on $C_{G}(A) / A$ is that of $\mathrm{SL}_{5}(2)$ on the skew-square of a 5-dimensional module $V_{5}(2)$;

(iv) A lies in no larger $z$-pure subgroup of $G$.

Proof. We have $Z_{0} \cong\left(Z_{2}\right)^{3}$, so $A \cong\left(Z_{2}\right)^{5}$. Moreover $A$ is $z$-pure by Step 2 . It is clear from Step 2 that any $z$-pure $\left(Z_{2}\right)^{5}$ in $F$ containing $Z_{0}$ is of the form $\left\langle Z_{0}, a_{1}^{\prime} \ldots a_{8}^{\prime}, b_{1}^{\prime} \ldots b_{8}^{\prime}\right\rangle$, and hence is conjugate in $F$ to $A$. Thus (i) is proved.

We next calculate $C_{G}(A)$. Since $C_{G}\left(Z_{0}\right)^{0}=F$, we have $C_{G}(A) \leqslant N_{G}(F)$. Moreover $N_{G}(F) / F C_{G}(F) \cong\left(Z_{2}\right)^{3} \cdot \operatorname{SL}_{3}(2)$, and $C_{G}\left(Z_{0}\right) / F$ lies in the normal subgroup $\left(Z_{2}\right)^{3}$ acting regularly on $\left\{J_{1}, \ldots, J_{8}\right\}$. It follows from (i) using a Frattini
argument that

$$
N_{G}(F)=F\left(N_{G}(A) \cap N_{G}(F)\right) .
$$

Moreover, $F \cap N_{G}(A)$ induces $S_{3}$ on the Klein group $A / Z_{0}$. Thus there is a subgroup $\langle r, s, t\rangle \leqslant N_{G}(A) \cap N_{G}(F)$ inducing the regular normal subgroup $\left(Z_{2}\right)^{3}$ on $\left\{J_{1}, \ldots, J_{8}\right\}$. Now there exist $i, j, k, l$ such that

$$
\left[r, a_{1} \ldots a_{8}\right]=\left(e_{i} e_{j}\right)^{s_{1}}, \quad\left[r, b_{1} \ldots b_{8}\right]=\left(e_{k} e_{l}\right)^{s_{2}},
$$

where $s_{1}, s_{2} \in\{0,1\}$. Replacing $r$ by $r\left(b_{i} b_{j}\right)^{s_{1}}\left(a_{k} a_{l}\right)^{s_{2}}$, we have $r \in C_{G}(A)$. Similarly we may take $s, t \in C_{G}(A)$, and so we have the subgroup

$$
\langle r, s, t\rangle \leqslant C_{G}(A) \text {. }
$$

Recall the set $\Delta$ of 4-sets defined in Step 2, and that for $R=\{i, j, k, l\} \in \Delta$, we set $a_{R}=a_{i} a_{j} a_{k} a_{l}$. Direct calculation gives

and hence

$$
C_{F}(A)=\left\langle A, e_{1}, a_{R}, b_{R} \mid R \in \Delta\right\rangle,
$$

$$
C_{G}(A)=\left\langle A, e_{1}, a_{R}, b_{R}, r, s, t \mid R \in \Delta\right\rangle .
$$


Thus $\left|C_{G}(A) / A\right|=2^{10}$ and $C_{G}(A) / A$ contains the elementary abelian subgroup $C_{F}(A) / A$ of order $2^{7}$.

We now show that $X=N_{G}(A) / C_{G}(A) \cong L_{5}(2)$. First note that for any $a \in A \backslash Z_{0}$, there is a maximal torus $T_{a}$ of $F$, and hence of $G$, such that $\left\langle Z_{0}, a\right\rangle \leqslant T_{a}$. Now $N_{G}\left(T_{a}\right) / T_{a} \cong W\left(E_{8}\right) \cong 2 . O_{8}^{+}(2)$. The non-trivial central element here is $w_{0}$, the longest element of $W\left(E_{8}\right)$, and the corresponding element $n_{w_{0}}$ of $N_{G}\left(T_{a}\right)$ inverts every element of $T_{a}$. Moreover, $A$ induces $\left\langle n_{w_{0}}\right\rangle$ on $T_{a}$. Write $Q=\Omega_{1}\left(O_{2}\left(T_{a}\right)\right) \cong\left(Z_{2}\right)^{8}$. Obviously $\left\langle Z_{0}, a\right\rangle \leqslant Q$, and $N_{G}\left(T_{a}\right)$ induces the group $O_{8}^{+}(2)$ acting naturally on $Q$. Since $\left\langle Z_{0}, a\right\rangle$ is $z$-pure, it is a totally singular 4-space in $Q$. Let $Y$ be any 3-space in $\left\langle Z_{0}, a\right\rangle$. Then there exists $g \in N_{G}\left(T_{a}\right)$ such that $Z_{0}^{g}=Y$. Further, $C_{G}(Y)^{0}=F^{g}$. Also $g$ normalizes $\left\langle T_{a}, n_{w_{0}}\right\rangle\left(=C_{G}(Q)\right)$, a group containing $A$, and hence $A \leqslant F^{g}$. Also $A^{g} \leqslant F^{g}$, and so by the uniqueness of the conjugacy class of $A^{g}$ in $F^{g}$ given by (i), there exists $f \in F^{g}$ such that $A^{f}=A^{g}$. Then

$$
g f^{-1} \in N_{G}(A), \quad Z_{0}^{g f^{-1}}=Y .
$$

As $a$ was an arbitrary element of $A \backslash Z_{0}$, we deduce that $X=N_{G}(A) / C_{G}(A)$ is transitive on the set of 3 -spaces in $A$. Moreover, the element $a_{1} a_{2}$ lies in $N_{G}(A)$ and induces a transvection in $X$. Consequently $X \cong L_{5}(2)$.

By $(*)$ there is an element $u \in N_{G}(A)$ of order 3 which acts on $\left\{J_{1}, \ldots, J_{8}\right\}$ as a product of two 3-cycles. Write $V_{1}=C_{F}(A) / A, V_{2}=C_{G}(A) / C_{F}(A)$. Elementary calculation shows that $\operatorname{dim} C_{V_{1}}(u)=3, \operatorname{dim} C_{V_{2}}(U)=1$, that $\left[V_{1}, u\right]$ is a direct sum of two 2-spaces, and that $\operatorname{dim}\left[V_{2}, u\right]=2$. Now the non-trivial irreducible modules in characteristic 2 for $X=L_{5}(2)$ of dimension 10 or less are the natural module $W$ of dimension 5, its dual $W^{*}$, and the skew-squares $\Lambda^{2} W, \Lambda^{2} W^{*}$. Since $\operatorname{dim}[W, u]=\operatorname{dim}\left[W^{*}, u\right]=2$, it follows from the above information that $C_{G}(A) / A$ must be the irreducible $X$-module $\Lambda^{2} W$ or $\Lambda^{2} W^{*}$ (and in particular that $\left.C_{G}(A) / A \cong\left(Z_{2}\right)^{10}\right)$. It is now immediate that $C_{G}(A)$ is a special group of order $2^{15}$, and so Parts (ii) and (iii) are proved.

It remains to prove (iv). Now $X=L_{5}(2)$ has precisely two orbits on the non-zero vectors of $\Lambda^{2} W$ or $\Lambda^{2} W^{*}$ (see $[20,2.5]$ ). In $C_{G}(A) / A$ these orbits are represented by $e_{1} A$ and $a_{R_{1}} b_{R_{2}} A$, where $R_{1}, R_{2} \in \Delta$ with $\left|R_{1} \cap R_{2}\right|=2$. Since $e_{1} \in e^{G}$ and $a_{R_{1}} b_{R_{2}}$ is an element of order 4 in $C_{G}(A)$, (iv) follows. This completes Step 7.

Recall now that $z=e_{1} e_{8} \in E$. Pick $z_{1} \in E \backslash\langle z\rangle$.

Step 8. We have $\left(C_{G}\left(z, z_{1}\right)\right)^{0}=S_{1} S_{2} \cong D_{4} D_{4}$, and $Z\left(S_{1}\right)=Z\left(S_{2}\right)=\left\langle z, z_{1}\right\rangle$. To see this, note that any $z$-pure Klein 4-subgroup $V$ of $G$ can be embedded in a maximal torus $T$ of $G$, and $N_{G}(T)$ induces $O_{8}^{+}(2)$ on the 8-space $\Omega_{1}\left(O_{2}(T)\right)$. Since it is $z$-pure, $V$ is a totally singular 2 -space here, and $O_{8}^{+}(2)$ is transitive on such 2 -spaces. Consequently $G$ has just one class of $z$-pure Klein 4-groups. Since $\left\langle z, z_{1}\right\rangle$ is one such, Step 8 follows.

Step 9. E contains no element interchanging $S_{1}$ and $S_{2}$. Suppose this is false, and pick $z_{2} \in E$ interchanging $S_{1}$ and $S_{2}$. Then $C_{G}\left(z, z_{1}, z_{2}\right)=\left\langle z, z_{1}, z_{2}\right\rangle N$ with $N \cong D_{4}$. Let $V=L(G)$, the 248-dimensional Lie algebra of $G$. Then $C_{V}\left(z, z_{1}, z_{2}\right)$ contains $L(N)$, so if $f=\operatorname{dim} C_{V}\left(z, z_{1}, z_{2}\right)$ then $f \geqslant 28$. On the other hand, by Step 
8, for each hyperplane $D_{0}$ of $\left\langle z, z_{1}, z_{2}\right\rangle$, we have, using [4, Corollary 9.2],

$$
\operatorname{dim} C_{V}\left(D_{0}\right)=\operatorname{dim}\left(L\left(D_{4}\right) \oplus L\left(D_{4}\right)\right)=56 .
$$

Thus by Proposition 2.16, $6 f+248=7 \times 56$. This yields $f=24$, a contradiction. This proves Step 9.

Step 10. There exists $z_{2} \in E$ such that $C_{G}\left(z, z_{1}, z_{2}\right)^{0}$ is conjugate to $F$. By Step 9, we have $E \leqslant S_{1} S_{2}=C_{G}\left(z, z_{1}\right)^{0}$. Thus for $z_{2} \in E \backslash\left\langle z, z_{1}\right\rangle$ the group $\left\langle z, z_{1}, z_{2}\right\rangle$ is contained in a maximal torus $T$ of $G$. Since $E$ is $z$-pure, this is a totally singular 3-subspace of $Q=\Omega_{1}\left(O_{2}(T)\right)$ regarded as an orthogonal space for $W^{Q}=O_{8}^{+}(2)$. Since $W$ is transitive on the set of totally singular 3 -spaces in $Q$, we deduce that $\left\langle z, z_{1}, z_{2}\right\rangle$ is conjugate to $\left\langle e_{i} e_{j}\right|$ all $\left.i, j\right\rangle=\left\langle Z(F) \cap z^{G}\right\rangle=Z_{0}$. Hence $C_{G}\left(z, z_{1}, z_{2}\right)^{0}$ is conjugate to $C_{G}\left(Z_{0}\right)^{0}=F$, as required for Step 10 .

By Step 10 we may take

$$
C_{G}\left(z, z_{1}, z_{2}\right)^{0}=F=J_{1} \ldots J_{8},
$$

and also $\left\langle z, z_{1}, z_{2}\right\rangle=Z_{0}$ and $E \leqslant N_{G}(F) \cap C_{G}(Z(F))$. Further, we may assume that

$$
J_{1} J_{2} J_{3} J_{8} \leqslant S_{1}, \quad J_{4} J_{5} J_{6} J_{7} \leqslant S_{2} .
$$

Step 11. We have $E \leqslant F$. Suppose this is false, and pick $x \in E \backslash F$. As $x \in z^{G}$ and also $x \in S_{1} S_{2}, \quad\langle x\rangle$ must act semiregularly on both $\left\{J_{1}, J_{2}, J_{3}, J_{8}\right\}$ and $\left\{J_{4}, J_{5}, J_{6}, J_{7}\right\}$. Clearly then there exists $R \in \Delta$ such that $x$ interchanges $\prod_{i \in R} J_{i}$ and $\prod_{i \notin R} J_{i}$. Consequently we can find $V \leqslant Z_{0}$ with $V \cong\left(Z_{2}\right)^{2}$ such that $C_{G}(V)=$ $R_{1} R_{2} \cong D_{4} D_{4}, \quad R_{1}$ contains $\prod_{i \in R} J_{i}, R_{2}$ contains $\prod_{i \notin R} J_{i}$, and such that $x$ interchanges $R_{1}$ and $R_{2}$. This cannot happen, by Step 9 .

Step 12. Completion of the proof for the group $G$. We have $Z_{0}=\left\langle z, z_{1}, z_{2}\right\rangle=$ $\left\langle e_{i} e_{j}\right|$ all $\left.i, j\right\rangle \leqslant E \leqslant F$. By Step 2, any conjugate of $z$ in $F \backslash Z(F)$ is of the form $a_{1} \ldots a_{8}$ or $a_{R} e_{r}$ with $R \in \Delta, r \notin R$. Since $\left\langle Z_{0}, a_{R} e_{r}\right\rangle$ contains elements of $e^{G}$, any element of $E \backslash Z_{0}$ must be of the form $a_{1} \ldots a_{8}$. Further, by Lemmas 2.3 and 2.5, $E$ must contain elements $a_{1} \ldots a_{8}$ and $b_{1} \ldots b_{8}$. Thus by Step 7(iv), we have

$$
E=A=\left\langle Z_{0}, a_{1} \ldots a_{8}, b_{1} \ldots b_{8}\right\rangle .
$$

All conclusions of the lemma for the algebraic group $G=E_{8}$ are now proved.

Step 13. Completion of the proof for the finite group $L$. We finally verify the statements of the lemma for the finite group $L=G_{\sigma}$. The group $E$ is contained in the $\sigma$-stable group $F=J_{1} \ldots J_{8}$. Since $\sigma$ centralizes $E$, it centralizes $Z_{0}=\left\langle e_{i} e_{j}\right|$ all $i, j\rangle$. It follows that $\sigma$ either fixes each $J_{i}$, or has four orbits of length 2 on the $J_{i}$. Correspondingly, $O^{p^{\prime}}\left(F_{\sigma}\right)$ is a central product of either eight copies of $\mathrm{SL}_{2}(q)$ or four copies of $\mathrm{SL}_{2}\left(q^{2}\right)$.

Suppose first that $O^{p^{\prime}}\left(F_{\sigma}\right)=\mathrm{SL}_{2}(q) \circ \ldots \circ \mathrm{SL}_{2}(q)$ (eight copies). If $E \leqslant O^{p^{\prime}}\left(F_{\sigma}\right)$ then $E$ is conjugate to a subgroup $E_{1}$ of $\mathrm{SL}_{2}(p) \circ \ldots \circ{ }^{\circ} \mathrm{SL}_{2}(p)$. Moreover we can argue as before that $N_{G}\left(E_{1}\right) \leqslant E_{8}(p)$ and that $E_{1}$ is unique up to $E_{8}(p)$ conjugacy. Thus $L=E_{8}(p)$ by the maximality of $M$, and the conclusion of the lemma holds in this case. Now assume that $E \$ O^{p^{\prime}}\left(F_{\sigma}\right)$. We may then take 
$a=a_{1} \ldots a_{8} \in O^{p^{\prime}}\left(F_{\sigma}\right)$ and $b=b_{1} \ldots b_{8} \notin O^{p^{\prime}}\left(F_{\sigma}\right)$. Now $M$ acts irreducibly on $E$ and $M / C_{M}(E) \leqslant \mathrm{SL}_{5}(2)$. Moreover $M / C_{M}(E)$ contains 2-elements such as $a_{1} a_{2}$, so $M / C_{M}(E)=\mathrm{SL}_{5}(2)$ (see [30]). Thus $N_{M}\left(Z_{0}\right)$ is transitive on $\left(E / Z_{0}\right)^{\#}$. But $N_{M}\left(Z_{0}\right)$ cannot send $a$ to $b$, since $a \in O^{p^{\prime}}\left(C\left(Z_{0}\right)\right)$ and $b \notin O^{p^{\prime}}\left(C\left(Z_{0}\right)\right)$. Hence this case does not occur.

Finally, suppose that $O^{p^{\prime}}\left(F_{\sigma}\right)=\mathrm{SL}_{2}\left(q^{2}\right) \circ \ldots \circ \mathrm{SL}_{2}\left(q^{2}\right)$ (four copies). As in the previous paragraph, the irreducibility of $M$ on $E$ and the existence of 2-elements in $M / C_{M}(E)$ imply that $M / C_{M}(E)=\mathrm{SL}_{5}(2)$. Consequently $N_{L}\left(Z_{0}\right)$ induces $\mathrm{SL}_{3}(2)$ on $Z_{0}$. This is impossible since $O^{p^{\prime}}\left(C_{L}\left(Z_{0}\right)\right)=O^{p^{\prime}}\left(F_{\sigma}\right)$, a central product of only four quasisimple groups, which cannot admit $\mathrm{SL}_{3}(2)$.

Lemma 2.17 completes the proof of Theorem 1, apart from the demonstration of the embedding $L_{4}(5)<E_{8}(4)$, which, as pointed out in the Introduction and in the proof of Lemma 2.10, is needed to show the non-maximality of the subgroup $5^{3} . \mathrm{SL}_{3}(5)$ in Table 1 when $p=2$. This embedding is exhibited in $\S 5$.

\section{Proof of Theorem 2}

In this section we give a proof of Theorem 2. Thus let $G$ be a simple exceptional adjoint algebraic group in characteristic $l$, and let $S$ be a subgroup of Aut $G$ such that $D=(S \cap G)^{0}$ is non-trivial, closed, and satisfies Conditions (1), (2) and (3) of Theorem 2. Suppose that $D$ is not parabolic or of maximal rank, that is, $D$ does not contain a maximal torus of $G$.

By Condition (2), the group $E=\Omega_{1}\left(O_{r}\left(C_{G}(D)\right)\right)$ is non-trivial. Clearly $S$ normalizes $E$, so by Condition (3) we have $D=C_{G}(E)^{0}$ and $N_{G}(D)=N_{G}(E)$. Let $Q=R_{u}(D)$. If $Q \neq 1$ or if $r=l$, then by [5,3.12], $S$ normalizes a parabolic subgroup $P$ containing $N(Q)$ or $N(E)$, forcing $D=P$; but then $D$ contains a maximal torus, a contradiction. Thus $Q=1$, and so $D$ is reductive. Moreover, if $S$ normalizes a connected subgroup $K \neq 1, G$ then by (3), $D=N(K)^{0}$, and hence $N(K)$ contains no maximal torus. In particular $Z(D)^{0}=1$, so $D$ is semisimple. As $S$ normalizes $D C(D)$, (3) gives $C(D)^{0} \leqslant D$, whence $C(D)$ is finite. Since $r \neq l, E$ consists of semisimple elements.

We claim next that if $1 \neq E_{1} \leqslant E$, then $C_{G}\left(E_{1}\right)$ contains no non-trivial normal torus. For otherwise, if $T \triangleleft C_{G}\left(E_{1}\right)$ with $T$ a non-trivial torus, then $D=C_{G}(E)^{0}$ normalizes $T$, and hence centralizes $T$, contrary to $C(D)$ being finite.

As $E$ lies in no torus, we see as in Lemma 2.4 that $r=2,3$ or 5 and $m(E) \geqslant 2$. Suppose that for some $e \in E^{\#}, C_{G}(e)$ is connected and has a factor $A_{n}$. Then since for semisimple $x \in A_{n} \backslash Z\left(A_{n}\right), C_{A_{n}}(x)$ has a normal torus, $C(E)$ must contain the factor $A_{n}$. But then $S$ normalizes the product of these factors $A_{n}$, and the normalizer of this product contains a maximal torus, a contradiction. This establishes the fact that for $e \in E^{\#}, C(e)$ has no normal torus, and if $C(e)$ is connected, it has no factor $A_{n}$. We conclude (cf. Lemma 2.6) that $C(e)$ is as follows:

\begin{tabular}{ccc}
\hline$G$ & $r$ & $C(e)$ \\
\hline$F_{4}$ & 2 & $B_{4}$ \\
$E_{0}$ & 3 & $\left(A_{2} \circ A_{2}^{\circ} A_{2}\right)\left\langle w_{3}\right\rangle$ \\
$E_{7}$ & 2 & $A_{7}\left\langle w_{2}\right\rangle$ \\
$E_{8}$ & 2 & $D_{8}$ \\
\hline
\end{tabular}


When $G=F_{4}$, we argue as in the last paragraph of the proof of Lemma 2.13 that $m(E) \leqslant 2$, whence $E$ lies in a maximal torus, a contradiction. For $G=E_{6}$, let $f \in E \backslash\langle e\rangle$. If $f \in C(e)^{0}$ then $C_{G}(e, f)$ has a normal torus, so $f \in C(e) \backslash C(e)^{0}$ and $E=\langle e, f\rangle$. We now obtain a contradiction as in the third paragraph of the proof of Lemma 2.11. When $G=E_{7}$, the same argument gives $E=\langle e, f\rangle$ with $f \in C(e) \backslash C(e)^{0}$. Then as in Lemma 2.15, $D=C(E)^{0}=D_{4}, N_{G}(D)=\left(2^{2} \times D_{4}\right) . S_{3}$. This is in the conclusion of Theorem 2. Finally, if $G=E_{8}$, we argue as in Steps 8-11 of Lemma 2.17 that $E \leqslant F=\left(A_{1}\right)^{8}$ and $E$ contains $Z_{0}=\left\langle Z(F) \cap z^{G}\right\rangle$. As $E$ does not lie in a torus, $E \neq Z_{0}$, so there exists $f \in E \backslash Z_{0}$. Then $C\left(Z_{0}, f\right)$ has a normal torus, which is a contradiction. This completes the proof of Theorem 2.

\section{Proof of Theorem 3}

Here we give the proof of Theorem 3. Thus let $G$ be an exceptional simple adjoint algebraic group in characteristic $l$, and let $A$ be an elementary abelian $r$-subgroup satisfying Conditions (a)-(e) of Theorem 3 . The proof runs parallel to that of Theorem 1 in $\S 2$. Since $N_{G}(A)$ is finite, it is clear that $A$ does not lie in a torus of $G$, and $C_{G}(A)^{0}=1$.

First, the proof of Lemma 2.4 gives

Lemma 4.1. (i) $A=\left\langle a^{G} \cap A\right\rangle$ for $a \in A^{\#}$.

(ii) $r$ is 2,3 or 5 , and $r=5$ only if $G=E_{8}$.

(iii) $m(A) \geqslant 2$; and $m(A) \geqslant 3$ if $(G, r)$ is not $\left(E_{6}, 3\right)$ or $\left(E_{7}, 2\right)$.

The fact that $C_{G}(A)^{0}=1$ implies the following analogue of Lemma 2.5.

Lemma 4.2. For $a \in A^{\#}, C_{G}(a)$ does not contain a central torus. Moreover, if $C_{G}(a)$ has a normal torus then $A \neq C_{G}(a)^{0}$.

Lemmas 4.1 and 4.2 give

Lemma 4.3. For $a \in A^{\#}$, the possibilities for $C_{G}(a)$ are those given in Table 3 of Lemma 2.6.

Lemma 4.4. (i) Suppose $|Z(\hat{G})|=r$. Then $\hat{E}$ is elementary abelian or extraspecial.

(ii) For $a \in A^{\#}, C_{G}(a)^{0}$ is semisimple.

Part (i) of Lemma 4.4 is proved as in Lemma 2.9(i). If (ii) fails, we argue as in Lemma 2.9 that $|A|=r^{2}$; but then $C_{G}(A)^{0} \neq 1$.

Now the proofs of Lemmas 2.10-2.17 give Theorem 3. Note that these proofs are sometimes greatly simplified by the hypothesis of Theorem 3, since $C_{G}(A)^{0}=1$ and the conclusions of Lemmas $2.1-2.3$ are subsumed by the much stronger hypothesis (e) of Theorem 3. 


\section{The embedding $L_{4}(5)<E_{8}(4)$}

In this final section we show that $E_{8}(4)$ contains a subgroup $L_{4}(5)$, and also that for $p \neq 2,5$, the algebraic group $E_{8}$ over the algebraic closure of $\mathbf{F}_{p}$ does not contain $L_{4}(5)$. As a consequence, the local subgroup $N(E) \cong 5^{3} \cdot \mathrm{SL}_{3}(5)$ of $E_{8}(4)$ constructed in Lemma 2.10 is non-maximal (see Table 1 of Theorem 1).

THEOREM 5.1. Let $G$ be the simple algebraic group of type $E_{8}$ over the algebraic closure of $\mathbf{F}_{p}$, where $p$ is prime and $p \neq 5$. Then $G$ contains a subgroup isomorphic to $L_{4}(5)$ if and only if $p=2$. Moreover, this embeds $L_{4}(5)$ in $E_{8}(4)$.

The subgroup $L_{4}(5)$ will be constructed as the group generated by two conjugates of the local subgroup $N_{G}(E) \cong 5^{3} \cdot \mathrm{SL}_{3}(5)$ constructed in Lemma 2.10. Write

$$
P=N_{G}(E) \text {. }
$$

We require a preliminary lemma concerning the group $P$.

Lemma 5.2. $P$ is a split extension of $E$ by $\mathrm{SL}_{3}(5)$.

Proof. Let $e \in E^{\#}$, and consider $C_{P}(e)$. This has the form $F \cdot \mathrm{SL}_{2}(5)$, where, in the notation of the proof of Lemma 2.10, $F=\left\langle a_{1}, b_{1}, a_{2}, b_{2}\right\rangle \cong 5^{1+4}$, an extraspecial group. By the construction of $E$ (Lemma 2.10), $C_{G}(e)=X_{1} X_{2}$ with each $X_{i} \cong \mathrm{SL}_{5}$. Since $C_{P}(e)$ normalizes each $X_{i}$, we conclude that $C_{P}(e) /\langle e\rangle$ acts completely reducibly on $F /\langle e\rangle$. Thus $F /\langle e\rangle$ has precisely six proper non-trivial $C_{P}(e) /\langle e\rangle$-invariant subgroups. Those lifting to extraspecial groups $5^{1+2}$ come in pairs (orthogonal complements under the action of $\mathrm{Sp}_{4}(5)$ on $F$ ). Since $E$ is abelian, $C_{P}(e)$ therefore normalizes a complement $\bar{E} /\langle e\rangle$ to $E /\langle e\rangle$ with $\bar{E}$ abelian. As $\mathrm{SL}_{2}(5)$ has trivial first cohomology on the natural 2-dimensional module, we have $\bar{E}=\langle e\rangle \times S$ with $S$ invariant under $\mathrm{SL}_{2}(5)$. It now follows that a Sylow 5-subgroup of $P$ splits over $E$, and hence $P$ splits, as required.

Proof of Theorem 5.1. In view of Lemma 5.2 we may write $P=E D$, with $D \cong \mathrm{SL}_{3}(5)$. Let $T \cong Z_{4} \times Z_{4}$ be a Cartan subgroup of $D$, and let $U$ be a Sylow 5-subgroup of $P$ such that $T \leqslant N_{P}(U)$ and $U=E(U \cap D)$. Consider the six $T$-composition factors of $U$. Each has centralizer $Z_{4}$ in $T$, and a direct check shows that these six subgroups of $T$ are distinct.

It follows that there are precisely six $T$-invariant subgroups of $U$ of order 5 . We call these positive root subgroups; thus a positive root subgroup is determined by its centralizer in $T$. The group $D$ contains three other $T$-invariant subgroups of order 5 . These are the usual root groups in $\mathrm{SL}_{3}(5)$ corresponding to negative roots. Each has centralizer in $T$ equal to that of some positive root group, and we call such a subgroup the opposite root group.

Label root subgroups as follows: $E=U_{\alpha} U_{\alpha+\beta} U_{\alpha+\beta+\gamma}$, with $\langle e\rangle=U_{\alpha+\beta+\gamma}$. Write $D=\left\langle U_{ \pm \beta}, U_{ \pm \gamma}\right\rangle$, where notation is chosen so that $S=U_{\gamma} U_{\beta+\gamma}$ (here $S$ is the group constructed in the proof of Lemma 5.2). The usual commutator relations hold among the root groups in $P$, this group being isomorphic to a maximal parabolic subgroup of $L_{4}(5)$. 
Now $\bar{E}=U_{\gamma} U_{\beta+\gamma} U_{\alpha+\beta+\gamma}$ (where $\bar{E}$ is as in Lemma 5.2). Consider $\bar{E}\left\langle U_{ \pm \beta}\right\rangle \leqslant$ $C_{G}(e)=X_{1} X_{2} \cong \mathrm{SL}_{5}{ }^{\circ} \mathrm{SL}_{5}$. The group $\left\langle U_{ \pm \beta}\right\rangle$ is diagonally embedded here (that is, it intersects each $X_{i}$ trivially), and it normalizes the intersection of $\bar{E}$ with each $X_{i}$. But $F$ intersects each $X_{i}$ in an extraspecial $5^{1+2}$, and the action of $\left\langle U_{ \pm \beta}\right\rangle$ on $\bar{E} /\langle e\rangle$ is irreducible. Consequently $\bar{E} \cap X_{i}=\langle e\rangle$ for $i=1,2$. From our construction of $E$ in Lemma 2.10, we thus see that $\bar{E}$ is conjugate to $E$.

Hence $\bar{P}=N_{G}(\bar{E}) \cong \bar{E} \cdot \mathrm{SL}_{3}(5)$, and there is a unique complement $\bar{D}$ to $\bar{E}$ which contains $T$. Then $\bar{D}$ contains precisely six $T$-invariant subgroups of order 5 , and we have $\bar{D}=\left\langle U_{ \pm \alpha}, U_{ \pm \beta}\right\rangle$. That is, we have a new root subgroup $U_{-\alpha}$, opposite to $U_{\alpha}$.

Set $M=\left\langle U_{ \pm \alpha}, U_{ \pm \beta}, U_{ \pm \gamma}\right\rangle$. We shall show that if $p=2$ then $M \cong L_{4}(5)$. To do this, it suffices to show that $\left[\left\langle U_{ \pm \gamma}\right\rangle, U_{-\alpha}\right]=1$; for then $\left[\left\langle U_{ \pm \alpha}\right\rangle,\left\langle U_{ \pm \gamma}\right\rangle\right]=1$ and an application of the Curtis-Tits relations [12, Theorem 1.4] implies that $M$ is an image of $L_{4}(5)$.

To this end, set $V=U_{\alpha+\beta} U_{\alpha+\beta+\gamma}, \quad \bar{V}=U_{\beta+\gamma} U_{\alpha+\beta+\gamma}$ and $R=V \bar{V} U_{\beta}=$ $U_{\alpha+\beta} U_{\alpha+\beta+\gamma} U_{\beta} U_{\beta+\gamma}$. We check that $R \cong 5^{4}$, and also $N_{P}(V)^{(\infty)}=R\left\langle U_{ \pm \gamma}\right\rangle$ and $N_{\bar{P}}(V)^{(\infty)}=R\left\langle U_{ \pm \alpha}\right\rangle$.

We now consider $N_{G}(R)$. Working in $C_{G}(e)$ we see that $C_{G}(V)^{0}$ is a maximal torus, say $H$, so $N_{P}(V) \leqslant N_{G}(H)$. As $N_{P}(V) / V$ is irreducible on $R / V$ and $5^{3}$ does not divide $\left|W\left(E_{8}\right)\right|$, we have $R \leqslant H$. Hence $N_{G}(R)$ normalizes $C_{G}(R)^{0}=H$. Consider the actions of $\left\langle U_{ \pm \alpha}\right\rangle$ and $\left\langle U_{ \pm \gamma}\right\rangle$ on $R$. We claim that these groups commute modulo $H$. To see this, note that $N_{G}(R)^{\prime}$ induces on $R$ an irreducible subgroup of $\mathrm{SL}_{4}(5)$ of order dividing $\left|W\left(E_{8}\right)\right|$, and containing a 5-element centralized by $\mathrm{SL}_{2}(5)$ (corresponding to the image of $U_{\alpha} \times\left\langle U_{ \pm \gamma}\right\rangle \leqslant P$ ). It follows that $N_{G}(R)^{\prime} / H \cong \mathrm{SL}_{2}(5) \circ \mathrm{SL}_{2}(5)$, corresponding to a subgroup $\Omega_{4}^{-}(2) \times \Omega_{4}^{-}(2)$ or $\Omega_{4}^{+}(4)$ in $\Omega_{8}^{+}(2)$. The former subgroup comes from $W\left(A_{4}\right) \times W\left(A_{4}\right)$, which does not act faithfully and irreducibly on $R$. Hence $N_{G}(R)^{\prime} / H$ corresponds to $\Omega_{4}^{+}(4)$.

Let $t$ be the involution in $T$ centralizing $\left\langle U_{ \pm \alpha}\right\rangle$ and $\left\langle U_{ \pm \gamma}\right\rangle$. Then $\langle t H\rangle=$ $Z(N(H) / H)$. Hence $C_{N(R)^{\prime}}(t)=\Omega_{1}\left(O_{2}(H)\right)$. $\left(\mathrm{SL}_{2}(5) \circ \mathrm{SL}_{2}(5)\right)$, showing that $\left\langle U_{ \pm \alpha}\right\rangle$ and $\left\langle U_{ \pm \gamma}\right\rangle$ commute modulo $\Omega_{1}\left(O_{2}(H)\right)$.

If $p=2$ then $O_{2}(H)=1$, so $\left\langle U_{ \pm \alpha}\right\rangle$ and $\left\langle U_{ \pm \gamma}\right\rangle$ commute, which implies that $M \cong L_{4}(5)$. Moreover, all the groups occurring in the proof exist in $E_{8}(4)$, so this embeds $L_{4}(5)$ in $E_{8}(4)$, as required.

It remains to show that if $p \neq 2,5$ then $G$ has no subgroup $L_{4}(5)$. To do this, we shall demonstrate that the extension $C_{N(R)}(t)=2^{8}$. $\left(\mathrm{SL}_{2}(5) \circ \mathrm{SL}_{2}(5)\right)$ does not split. This implies that $\left\langle U_{ \pm \alpha}\right\rangle$ and $\left\langle U_{ \pm \gamma}\right\rangle$ do not commute. Hence if $G$ had a subgroup $L_{4}(5)$, we could have performed all the above calculations within this subgroup to obtain a contradiction.

Thus suppose for a contradiction that the extension splits. We have $C_{N(R)^{\prime}}(t)=$ $2^{9}$. $\left(\mathrm{Alt}_{5} \times \mathrm{Alt}_{5}\right)$ with central involution $t$. This is a subgroup of $C_{G}(t)=D_{8}$. Let $\bar{N} \cong 2^{8}$ be the image of the normal $2^{9}$ in $C_{G}(t) /\langle t\rangle=P \Omega_{16}(K)$, where $K=\overline{\mathbb{F}}_{p}$. Let $N$ be the preimage of $\bar{N}$ in $\Omega_{16}(K)$, and let $C$ be the preimage of $C_{N(R)^{\prime}}(t) /\langle t\rangle$ in $\Omega_{16}(K)$.

We claim that $N$ is extraspecial. Otherwise, it is abelian (since the top factor $\mathrm{Alt}_{5} \times \mathrm{Alt}_{5}$ acts irreducibly on $\bar{N}$ ), and an easy argument using the action of elements of order 5 shows that $N=N_{1} \times\langle z\rangle$, where $z$ is the central involution in $\Omega_{16}(K)$ and $N_{1}$ is normal in $C$. The action of $C$ on $N_{1}$ is $\Omega_{4}^{+}(4)$, which has orbits of size 75 and 180 on the linear characters of $N_{1}$, and hence by Clifford's theorem, $C$ cannot lie in $\Omega_{16}(K)$, a contradiction. Thus $N$ is extraspecial, as claimed. 
We now use an argument of R. L. Griess to complete the proof. We are assuming that the extension $C=N . \Omega_{4}^{+}(4)$ splits. Take a subgroup $A$ of type $\Omega_{3}(4)$. There are an involution $a \in A$ and a singular vector $s \in N /\langle z\rangle$ for which $s^{a}=s+n$, where $n$ is a non-singular vector fixed by $A$ and $n$ lifts to an element of order 4 in $N$. In the group $N$ the preimage of $\langle s, n\rangle$ is a dihedral group with $a$ inverting the element of order 4 , which corresponds to $n$. But $A$ normalizes this $Z_{4}$ and $A=A^{\prime}$. This is a contradiction, completing the proof of Theorem 5.1.

\section{References}

1. A. V. AlEKSEEVSKII, 'Finite commutative Jordan subgroups of complex simple Lie groups', Funct. Anal. Appl. 8 (1974) 277-279.

2. M. AschBACHER, 'On finite groups of Lie type and odd characteristic', J. Algebra 66 (1980) 400-424.

3. M. Aschbacher and G. M. Seitz, 'Involutions in Chevalley groups over fields of even order', Nagoya Math. J. 63 (1976) 1-91.

4. A. BOREL, Linear algebraic groups (Benjamin, New York, 1969).

5. A. BOREL and J. Trrs, 'Éléments unipotents et sous-groupes paraboliques des groupes réductifs', Invent. Math. 12 (1971) 95-104.

6. A. V. Borovik, 'Jordan subgroups and orthogonal decompositions', Algebra i Logika 28 (1989) 372-383 (in Russian).

7. A. V. BoROVIK, 'The structure of finite subgroups of simple algebraic groups', Algebra i Logika 28 (1989) 249-279 (in Russian).

8. N. BuRgoyne and C. Williamson, 'On a theorem of Borel and Tits for finite Chevalley groups', Arch. Math. 27 (1976) 489-491.

9. R. W. CARTER, Simple groups of Lie type (John Wiley, London, 1972).

10. R. W. CARTER, 'Conjugacy classes in the Weyl group', Compositio Math. 25 (1972) 1-59.

11. J. H. Conway, R. T. Curtis, S. P. Norton, R. A. Parker, and R. A. Wilson, Atlas of finite groups (Clarendon Press, Oxford, 1985).

12. C. W. CuRTis, 'Central extensions of groups of Lie type', J. Reine Angew. Math. 220 (1965) 174-185.

13. D. I. Deriziotis, 'The centralizers of semisimple elements of the Chevalley groups $E_{7}$ and $E_{8}$ ', Tokyo J. Math. 6 (1983) 191-216.

14. D. I. Deriziotis and M. W. Liebeck, 'Centralizers of semisimple elements in finite twisted groups of Lie type', J. London Math. Soc. (2) 31 (1985) 48-54.

15. D. GoRENSTEIn, Finite groups (Harper and Row, New York, 1968)

16. D. GORENSTEIN and R. LyONS, 'The local structure of finite groups of characteristic 2 type', Mem. Amer. Math. Soc. 42 (1983)

17. N. IWAHORI, 'Centralizers of involutions in finite Chevalley groups', Seminar on algebraic groups and related finite groups (eds A. Borel et al), Lecture Notes in Mathematics 131 (Springer, Berlin, 1970), pp. 267-295.

18. P. B. Kleidman, 'The maximal subgroups of the Steinberg triality groups ${ }^{3} D_{4}(q)$ and of their automorphism groups', J. Algebra 115 (1988) 182-199.

19. A. I. Kostrikin, 'Invariant lattices in Lie algebras and their automorphism groups', Group theory, Proceedings of the 1987 Singapore Conference (eds K. N. Cheng and Y. K. Leong, de Gruyter, New York, 1989), pp. 171-181.

20. M. W. LIEBECK, 'The affine permutation groups of rank three', Proc. London Math. Soc. (3) 54 (1987) $477-516$

21. M. W. Liebeck, 'The local maximal subgroups of the finite simple groups', Proc. Sympos. Pure Math. 47 (1987) 455-461.

22. M. W. LiEBECK and G. M. Sertz, 'Maximal subgroups of exceptional groups of Lie type, finite and algebraic', Geom. Dedicata 36 (1990) 353-387.

23. M. W. LiebeCK, J. SAXl, and G. M. Seirz, 'Subgroups of maximal rank in groups of Lie type', Proc. London Math. Soc., to appear.

24. J. MCLaughlin, 'Some groups generated by transvections', Arch. Math. 18 (1967) 364-368.

25. S. P. NORTON and R. A. Wilson, 'The maximal subgroups of $F_{4}(2)$ ', Comm. Algebra 17 (1989) 2809-2824.

26. T. SPRinger and R. STEINBERg, 'Conjugacy classes', Seminar on algebraic groups and related finite groups (eds A. Borel et al), Lecture Notes in Mathematics 131 (Springer, Berlin, 1970), pp. $168-266$.

27. R. SternberG, 'Endomorphisms of linear algebraic groups', Mem. Amer. Math. Soc. 80 (1968). 
30. A. WAGNER, 'The subgroups of $\mathrm{PSL}_{5}\left(2^{a}\right)$ ', Resultate Math. 1 (1978) 207-226.

31. H. N. WARD, 'On Ree's series of simple groups', Trans. Amer. Math. Soc. 121 (1966) 62-89.

\author{
Arjeh M. Cohen \\ CWI \\ Kruislaan 413 \\ 1098 SJ Amsterdam \\ The Netherlands
}

\section{Jan Saxl}

Gonville and Caius College

Cambridge CB2 1TA

\author{
Martin W. Liebeck \\ Department of Mathematics \\ Imperial College \\ Huxley Building \\ 180 Queen's Gate \\ London SW7 2BZ
}

Gary M. Seitz

Department of Mathematics University of Oregon

Eugene

Oregon 97403

U.S.A. 